\title{
On the nature of the fourth generation neutrino and its implications
}

\author{
Alberto Aparici;, Juan Herrero-Garcíał Nuria Rius ${ }^{\ddagger}$ and Arcadi Santamaria ${ }^{\S}$ \\ Dept. de Física Teórica, and IFIC, Universidad de Valencia-CSIC \\ Edificio de Institutos de Paterna, Apt. 22085, 46071 Valencia, Spain
}

\begin{abstract}
We consider the neutrino sector of a Standard Model with four generations. While the three light neutrinos can obtain their masses from a variety of mechanisms with or without new neutral fermions, fourth-generation neutrinos need at least one new relatively light right-handed neutrino. If lepton number is not conserved this neutrino must have a Majorana mass term whose size depends on the underlying mechanism for lepton number violation. Majorana masses for the fourth-generation neutrinos induce relative large two-loop contributions to the light neutrino masses which could be even larger than the cosmological bounds. This sets strong limits on the mass parameters and mixings of the fourth-generation neutrinos.
\end{abstract}

Keywords: Neutrino Physics, Beyond Standard Model.

*alberto.aparici@uv.es

†juan.a.herrero@uv.es

${ }^{\ddagger}$ nuria@ific.uv.es

$\S$ arcadi.santamaria@uv.es 


\section{Contents}

1. Introduction 1

2. Light neutrino masses 3

2.1 Dirac masses 3

2.2 Seesaw 3

2.2.1 Type I: fermionic singlets 3

2.2.2 Type II: scalar triplet 4

2.2.3 Type III: fermionic triplets 5

2.3 Others 6

2.4 Weinberg operator 6

$\begin{array}{lll}\text { 3. Fourth-generation neutrino masses } & 7\end{array}$

4. Light neutrino masses induced by new generations 11

5. Phenomenological constraints $\quad \mathbf{1 5}$

$\begin{array}{lll}5.1 & \text { Direct searches } & 15\end{array}$

$\begin{array}{ll}5.2 \text { Lepton flavour violation } & 16\end{array}$

$\begin{array}{ll}5.3 \text { Universality tests } & 17\end{array}$

$\begin{array}{lll}5.4 & \text { Light neutrino masses } & 18\end{array}$

$\begin{array}{lll}5.5 & \text { Neutrinoless double beta decay }(0 \nu 2 \beta) & 19\end{array}$

5.6 Four generations and the Higgs boson 21

6. Conclusions 22

$\begin{array}{ll}\text { 7. Acknowledgments } & 23\end{array}$

A. A model for calculable right-handed neutrino masses 23

\section{Introduction}

In the framework of the Standard Model (SM), fermions are grouped into three families, each containing a doublet of quarks and a doublet of leptons. The number of families is not a constructive parameter of the theory, and it could well be four or more; for this reason, the enlargement of the SM with new generations has been commonly considered [1], and it has proven to help in dealing with several problems, such as the lack of $\mathrm{CP}$ violation in the SM to explain the baryon asymmetry of the universe [2] or the structure of the leptonic mass matrices [3]. The currently available SM observables, however, constrain quite tightly 
the properties of such new families [4], and the global electroweak fits seem to disfavour a scenario with more than five generations $[5,6]$; maybe the most striking result against the existence of additional families is the LEP measurement of the number of neutrinos at the $Z$ peak, which forbids more than three light neutrinos [4], but even this can be dodged if the neutrinos of the new generations are too heavy to be produced in $Z$ decays. All in all, the existence of new generations is not actually excluded, and it seems worth being considered [1], even more now that the LHC is working and exploring the relevant mass range.

On the other hand, right-handed neutrinos constitute a common new physics proposal, usually linked to the generation of neutrino masses. This is particularly interesting nowadays, ever since we gathered compelling evidence that neutrinos do have masses, that they lie well below the other fermions' ones, and that their mixing patterns differ extraordinarily from those of the quark sector (for a review on the matter of neutrino masses see, for example, [7]). The most straightforward way to construct a mass term for the neutrinos within the SM is just to rely on the Higgs mechanism, and so to write the corresponding Yukawa couplings; for that aim, one needs some fermionic fields which carry no SM charge: right-handed neutrinos. However, we do not know whether neutrinos are Dirac or Majorana. If they are Dirac, the smallness of the neutrino mass scale remains unexplained, for it would be just a product of the smallness of the corresponding Yukawa couplings. In order to provide such an explanation, many models and mechanisms have been proposed: in the so-called see-saw models, the lightness of the neutrino mass scale is a consequence of the heaviness of another scale. For instance, this scale is the lepton-number-violating (LNV) Majorana mass of the extra right-handed neutrinos in type I see-saw [8-11]. On the other hand, radiative models propose that neutrino masses are originated via suppressed, high-order processes [12-15]. Although some of these proposals do not require right-handed neutrinos, for the sake of generality it is a good idea to consider their possible involvement in the generation of neutrino masses.

In this work we aim to discuss the naturality of the various scenarios arising when new generations and right-handed neutrinos are brought together. Several previous works have considered such association, either explicitly, in order to provide a mechanism for mass generation, or implicitly, when assuming Dirac neutrinos in their analyses [16-25]. We argue that unless a symmetry is invoked which separates the new family from the first three, the coexistence of both Dirac and Majorana neutrinos is not stable under radiative corrections and doesn't seem natural $[25,26]$. Furthermore, the presence of a fourth family plus a right-handed Majorana neutrino triggers the generation of Majorana masses for the light species through a well-known mechanism [16,17,23,25,27-29]; the upper bounds on the light neutrino masses can thus be translated into bounds on the mixings with the new, heavy generations.

This paper is structured as follows. In section 2 we start by reviewing the different mechanisms which can provide light neutrino masses. In section 3 we discuss the naturalness of those mechanisms to generate the fourth-family neutrino mass and conclude that at least one right-handed neutrino is needed. Assuming that light neutrinos are Majorana, we use naturalness arguments to provide a lower bound on the Majorana mass of the right- 
handed neutrino. In section 4 we consider a minimal four generation SM with only one relatively light right-handed neutrino and Majorana masses for light neutrinos parametrized by the Weinberg operator $[30,31]$. We describe the radiative, two-loop contribution of the heavy fourth-family neutrinos to the light neutrino mass matrix. In section 5 we discuss the phenomenological consequences of this minimal four-generation scenario with heavy Majorana neutrinos (lepton flavour violation, universality bounds, light neutrino masses, neutrinoless double beta decay,...), and we conclude in section 6. Appendix A is devoted to describe an explicit example in which a finite Majorana mass for the fourth-generation right-handed neutrino is radiatively generated.

\section{Light neutrino masses}

The huge hierarchy between neutrino masses and those of all other fermions has triggered the appearance of many different mechanisms to explain the lightness of neutrinos. Here we briefly review some of these mechanisms, with special emphasis on the frameworks that are able to explain neutrino masses including a fourth generation, which will be discussed in the next section.

\subsection{Dirac masses}

If there are right-handed neutrinos and a conserved global symmetry (for instance $B-L$ ) prevents them from having a Majorana mass, neutrinos are Dirac particles, as all other fermions in the SM. However, in this scenario there is no explanation for the smallness of neutrino masses, having to impose by hand extremely tiny Yukawa couplings, approximately 6 (11) orders of magnitude smaller than the electron (top) one. Therefore, although in principle it is possible, a Dirac nature does not seem the most natural option for neutrinos (but see, for example, [3], for a proposal in this direction which avoids tiny Yukawas).

\subsection{Seesaw}

Seesaw models are minimal extensions of the SM which can naturally lead to tiny (Majorana) neutrino masses, keeping the SM gauge symmetry, $S U(3)_{\mathrm{C}} \otimes S U(2)_{\mathrm{L}} \otimes U(1)_{\mathrm{Y}}$ and renormalizability, but giving up the (accidental) lepton number conservation of the SM. Let's explain briefly the different types to fix notation.

\subsubsection{Type I: fermionic singlets}

In type I see-saw [8-11,32], $n$ SM fermionic singlets with zero hypercharge are added to the SM; these have the quantum numbers of right-handed neutrinos, and can be denoted by $\nu_{\mathrm{R} i}$. Note that to explain neutrino data, which requires al least two massive neutrinos, a minimum of two extra singlets are needed. Having no charges under the SM, Majorana masses for right-handed neutrinos are allowed by gauge invariance, so the new terms in the Lagrangian are:

$$
\mathcal{L}_{\nu_{\mathrm{R}}}=i \overline{\nu_{\mathrm{R}}} \gamma^{\mu} \partial_{\mu} \nu_{\mathrm{R}}-\left(\frac{1}{2} \overline{\nu_{\mathrm{R}}^{\mathrm{c}}} M \nu_{\mathrm{R}}+\bar{\ell} \tilde{\phi} Y \nu_{\mathrm{R}}+\text { H.c. }\right)
$$


where $\ell$ and $\phi$ are respectively the lepton and Higgs SM doublets, $\tilde{\phi}=i \tau_{2} \phi^{*}$ with $\tau_{2}$, the second Pauli matrix, acting on the $S U(2)_{\mathrm{L}}$ indices, $M$ is a $n \times n$ symmetric matrix, $Y$ is a general $3 \times n$ matrix and we have omitted flavour indices for simplicity. After spontaneous symmetry breaking (SSB), $\langle\phi\rangle=v_{\phi}$ with $v_{\phi}=174 \mathrm{GeV}$, the neutrino mass terms are given by

$$
\mathcal{L}_{\nu \text { mass }}=-\frac{1}{2}\left(\overline{\nu_{\mathrm{L}}} \overline{\nu_{\mathrm{R}}^{\mathrm{c}}}\right)\left(\begin{array}{cc}
0 & m_{\mathrm{D}} \\
m_{\mathrm{D}}^{\mathrm{T}} & M
\end{array}\right)\left(\begin{array}{c}
\nu_{\mathrm{L}}^{\mathrm{c}} \\
\nu_{\mathrm{R}}
\end{array}\right)+\text { H.c. },
$$

where $m_{D}=Y v_{\phi}$. The mass scale for right-handed neutrinos is in principle free, however if $M \gg m_{\mathrm{D}}$, upon block-diagonalization one obtains $n$ heavy leptons which are mainly $\mathrm{SM}$ singlets, with masses $\sim M$, and the well-known see-saw formula for the effective light neutrino Majorana mass matrix,

$$
m_{\nu} \simeq-m_{\mathrm{D}} M^{-1} m_{\mathrm{D}}^{\mathrm{T}}
$$

which naturally explains the smallness of light neutrino masses as a consequence of the presence of heavy SM singlet leptons.

\subsubsection{Type II: scalar triplet}

The type II see-saw [33-37] only adds to the SM field content one scalar triplet with hypercharge $Y=1$ (we adopt the convention that $Q=Y+T_{3}$ ) and assigns to it lepton number $L=-2$. In the doublet representation of $S U(2)_{\mathrm{L}}$ the triplet can be written as a $2 \times 2$ matrix, whose components are

$$
\chi=\left(\begin{array}{cc}
\chi^{+} / \sqrt{2} & \chi^{++} \\
\chi_{0} & -\chi^{+} / \sqrt{2}
\end{array}\right)
$$

Gauge invariance allows a Yukawa coupling of the scalar triplet to two lepton doublets,

$$
\mathcal{L}_{\chi}=\left(\left(Y_{\chi}^{\dagger}\right)_{\alpha \beta} \overline{\tilde{\ell}}_{\alpha} \chi \ell_{\beta}+\text { H.c. }\right)-V(\phi, \chi)
$$

where $Y_{\chi}$ is a symmetric matrix in flavour space, and $\tilde{\ell}=i \tau_{2} \ell^{\mathrm{c}}$. The scalar potential has, among others, the following terms:

$$
V(\phi, \chi)=m_{\chi}^{2} \operatorname{Tr}\left[\chi \chi^{\dagger}\right]-\left(\mu \tilde{\phi}^{\dagger} \chi^{\dagger} \phi+\text { H.c. }\right)+\ldots
$$

The $\mu$ coupling violates lepton number explicitly, and it induces a vacuum expectation value $(\mathrm{VEV})$ for the triplet via the $\mathrm{VEV}$ of the doublet, even if $m_{\chi}>0$. In the limit $m_{\chi} \gg v_{\phi}$ this VEV can be approximated by:

$$
\langle\chi\rangle \equiv v_{\chi} \simeq \frac{\mu v_{\phi}^{2}}{m_{\chi}^{2}}
$$

then, the Yukawa couplings in equation (2.5) lead to a Majorana mass matrix for the left-handed neutrinos

$$
m_{\nu}=2 Y_{\chi} v_{\chi}=2 Y_{\chi} \frac{\mu v_{\phi}^{2}}{m_{\chi}^{2}} .
$$


Neutrino masses are thus proportional to both $Y_{\chi}$ and $\mu$. Such dependence can be understood from the Lagrangian, since the breaking of lepton number $L$ results from the simultaneous presence of the Yukawa and $\mu$ couplings. As long as $m_{\chi}^{2}$ is positive and large, $v_{\chi}$ will be small, in agreement with the constraints from the $\rho$ parameter, $v_{\chi} \lesssim 6 \mathrm{GeV}[38]{ }^{1}$ Moreover, the parameter $\mu$, which has dimensions of mass, can be naturally small, because in its absence lepton number is recovered, increasing the symmetry of the model.

\subsubsection{Type III: fermionic triplets}

In the type III see-saw model $[40,41]$, the $\mathrm{SM}$ is extended by fermion $S U(2)_{\mathrm{L}}$ triplets $\Sigma_{\alpha}$ with zero hypercharge. As in type I, at least two fermion triplets are needed to have two non-vanishing light neutrino masses. We choose the spinors $\Sigma_{\alpha}$ to be right-handed under Lorentz transformations and write them in $S U(2)$ Cartesian components $\vec{\Sigma}_{\alpha}=$ $\left(\Sigma_{\alpha}^{1}, \Sigma_{\alpha}^{2}, \Sigma_{\alpha}^{3}\right)$. The Cartesian components can be written in terms of charge eigenstates as usual

$$
\Sigma_{\alpha}^{+}=\frac{1}{\sqrt{2}}\left(\Sigma_{\alpha}^{1}-i \Sigma_{\alpha}^{2}\right), \quad \Sigma_{\alpha}^{0}=\Sigma_{\alpha}^{3}, \quad \Sigma_{\alpha}^{-}=\frac{1}{\sqrt{2}}\left(\Sigma_{\alpha}^{1}+i \Sigma_{\alpha}^{2}\right)
$$

and the charged components can be further combined into negatively charged Dirac fermions $E_{\alpha}=\Sigma_{\alpha}^{-}+\Sigma_{\alpha}^{+c}$. Using standard four-component notation the new terms in the Lagrangian are given by

$$
\mathcal{L}_{\Sigma}=i \overline{\vec{\Sigma}_{\alpha}} \gamma^{\mu} D_{\mu} \cdot \vec{\Sigma}_{\alpha}-\left(\frac{1}{2} M_{\alpha \beta} \overline{\vec{\Sigma}_{\alpha}^{c}} \cdot \vec{\Sigma}_{\beta}+Y_{\alpha \beta} \overline{\ell_{\alpha}}\left(\vec{\tau} \cdot \vec{\Sigma}_{\beta}\right) \tilde{\phi}+\text { H.c. }\right),
$$

where $Y$ is the Yukawa coupling of the fermion triplets to the SM lepton doublets and the Higgs, and $M$ their Majorana mass matrix, which can be chosen to be diagonal and real in flavour space.

After SSB the neutrino mass matrix can be written as

$$
\mathcal{L}_{\nu \text { mass }}=-\frac{1}{2}\left(\overline{\nu_{\mathrm{L}}} \overline{\Sigma^{0 \mathrm{c}}}\right)\left(\begin{array}{cc}
0 & m_{\mathrm{D}} \\
m_{\mathrm{D}}^{\mathrm{T}} & M
\end{array}\right)\left(\begin{array}{c}
\nu_{\mathrm{L}}^{\mathrm{c}} \\
\Sigma^{0}
\end{array}\right)+\text { H.c. }
$$

which is the same as in the type I see-saw just replacing the singlet right-handed neutrinos by the neutral component of the triplets, $\Sigma_{\alpha}^{0}$, and therefore leads to a light neutrino Majorana mass matrix

$$
m_{\nu} \simeq-m_{\mathrm{D}} M^{-1} m_{\mathrm{D}}^{\mathrm{T}}
$$

However, since the triplet has also charged components with the same Majorana mass, in this case there are stringent lower bounds on the new mass scale, $M \gtrsim 100 \mathrm{GeV}$.

\footnotetext{
${ }^{1}$ This bound is calculated after the inclusion of the one-loop corrections to the $\rho$ parameter, and is slightly looser than other previously obtained from electroweak global fits (see, for example, [39]). Note also that the authors of [38] use a different normalisation for the VEV, and hence the difference between their value and the one we present here.
} 


\subsection{Others}

Here we briefly summarize non minimal mechanisms which also lead to Majorana light neutrino masses. Most of these models do not include right-handed neutrinos and are designed to obtain tiny Majorana masses for the left-handed SM neutrinos, so we can anticipate that they will not be appropriate for the fourth generation.

a) Radiative mechanisms. Small Majorana neutrino masses may also be induced by radiative corrections [12-15]. Typically, on top of loop factors of at least $1 /(4 \pi)^{2}$, there are additional suppressions due to couplings or ratios of masses, leading to the observed light neutrino masses with a new physics scale not far above the electroweak one.

b) Supersymmetry. There is an intrinsically supersymmetric way of breaking lepton number by breaking the so-called $\mathrm{R}$ parity [42-51] (for a review see [52]). In this scenario, the SM doublet neutrinos mix with the neutralinos, i.e., the supersymmetric (fermionic) partners of the neutral gauge and Higgs bosons. As a consequence, Majorana masses for neutrinos (generated at tree level and at one loop) are naturally small because they are proportional to the small R-parity-breaking parameters.

\subsection{Weinberg operator}

As we have mentioned, it does not seem very natural that neutrinos are Dirac particles; assuming that they are Majorana, we will be often interested in abstracting from the actual mechanism of mass generation. In such case, if the light degrees of freedom are those of the SM we can parametrise the Majorana masses in terms of the well-known dimension 5 Weinberg operator ${ }^{2}[30,31]$ :

$$
\mathcal{L}_{5}=\frac{1}{2} \frac{c_{\alpha \beta}}{\Lambda_{W}}\left(\overline{\ell_{\alpha}} \tilde{\phi}\right)\left(\phi^{\dagger} \tilde{\ell}_{\beta}\right)+\text { H.c. },
$$

where $\Lambda_{W} \gg v_{\phi}$ is the scale of new physics and $c_{\alpha \beta}$ are model-dependent coefficients with flavour structure, which in some models can carry additional suppression due to loop factors (as is the case in radiative mechanisms) and/or ratios of mass parameters (for instance in type II see-saw $\left.c \propto \mu / m_{\chi}\right)$. In those cases we will assume that $\Lambda_{W}$ is directly related to the masses of the new particles and absorb all suppression factors in $c_{\alpha \beta}$.

Upon electroweak symmetry breaking, the Weinberg operator leads to a Majorana mass matrix for the light neutrinos of the form

$$
m_{\nu}=c \frac{v_{\phi}^{2}}{\Lambda_{W}} .
$$

Notice that if $c_{\alpha \beta}$ is suppressed, the scale $\Lambda_{W}$ does not need to be extremely large in order to fit light neutrino masses and, thus, the Weinberg operator can parametrize a variety of Majorana neutrino mass models, including those with masses generated radiatively.

\footnotetext{
${ }^{2}$ In supersymmetric models, $\tilde{\phi}=H_{u}$, since there are two Higgs doublets.
} 


\section{Fourth-generation neutrino masses}

If there exists a fourth generation, the fourth-generation neutrinos must be massive (with masses $\gtrsim m_{Z} / 2$ in order to avoid the strong limits for the number of active neutrinos found at LEP). In principle all mass mechanisms available for the light neutrinos are also available to the fourth-generation neutrinos, however, the fact that they must be quite massive changes completely the discussion of the naturalness of the different mechanisms. Let us discuss them:

\section{a) Dirac masses.}

Since the fourth generation must be at the electroweak scale, this mechanism of mass generation is quite natural for the fourth-generation neutrinos as long as lepton number is conserved.

\section{b) Fermionic singlets with Majorana mass.}

If lepton number is not conserved there is no reason to forbid a Majorana mass term for right-handed neutrinos (see the discussion below). However if $m_{R} \gg m_{D}$ the see-saw formula applies and the spectrum contains a relatively light, almost-active neutrino with mass $m_{4} \sim m_{D}^{2} / m_{R}$, which must be heavier than $m_{Z} / 2$. Therefore $m_{R}<m_{D}^{2} / m_{Z}$ and the mass of the right-handed neutrino cannot be much larger than the electroweak scale. On the other hand, if $m_{R} \ll m_{D}$ there are two almost degenerate neutrinos and we are in the pseudo-Dirac limit, which does not pose any problem.

\section{c) Scalar triplet.}

In principle, as in the case of light neutrinos, scalar triplets could also be used to obtain Majorana masses for the fourth-generation neutrinos. However, the strong limits on the triplet's VEV coming from the $\rho$ parameter $v_{\chi} \lesssim 6 \mathrm{GeV}$ will yield fourth-generation neutrino masses too small. This limit could be relaxed a bit if radiative corrections to the $\rho$ parameter coming from triplet masses are large and such that cancel in part the deviations induced by the triplet's VEV, but this will require quite a high degree of fine tuning among rather different quantities. Therefore, this mechanism alone is not a natural mechanism for the fourth-generation neutrino masses.

\section{d) Fermionic triplets.}

This is similar to b), but together with the right-handed neutrinos there come new charged fermions degenerate with the neutral component. Since production limits tell us that the charged fermions must be heavier than about $100 \mathrm{GeV}$, in this case the pseudo-Dirac limit is not possible. Moreover these new fermions cannot be extremely heavy, because otherwise the active neutrino will be too light. We conclude that this mechanism is viable but much more constrained than $\mathrm{b}$ ). 


\section{e) Radiative mechanisms and SUSY with broken R parity.}

Neutrino masses in these models are strongly suppressed with respect to the electroweak scale by either loop factors, couplings and/or ratios of masses. Therefore they are not viable for the fourth generation.

\section{f) Weinberg operator.}

In principle the Weinberg operator could also be used to give Majorana masses to the fourth-generation neutrinos. However, it will provide masses $\mathcal{O}\left(v_{\phi}^{2} / \Lambda_{W}\right)$ which should be $\gtrsim m_{Z} / 2$, so the scale of new physics $\Lambda_{W}$ can not be much larger than the electroweak scale $v_{\phi}$ and the effective theory does not make sense. Therefore the Weinberg operator does not provide a useful parametrization of the fourth-generation neutrino mass.

We can therefore conclude that only a), b) (which includes a) in some limit) and possibly d) are good mechanisms for the fourth-generation neutrino masses. It seems then that to describe correctly the fourth-generation neutrino one needs at least one righthanded neutrino (either SM singlet or triplet) which has standard Dirac couplings to the doublets. If this $\mathrm{RH}$ neutrino is a SM triplet, we have seen that its Majorana mass is in the range $100 \mathrm{GeV} \lesssim m_{R} \lesssim$ few $\mathrm{TeV}$.

However, if the right-handed neutrino is a SM singlet it could have a very small or even vanishing Majorana mass term. Is it natural to have Dirac neutrinos for the fourth generation? The answer is simple: yes, provided there is a symmetry that protects them from acquiring a Majorana mass term. This is not the situation if the light neutrinos are Majorana, as most of the SM extensions that we considered in section 2, and they can mix freely with the heavy fourth family. We argue that in such a case a Majorana mass term for the fourth right-handed neutrino should be allowed just on symmetry grounds, and in fact, based on naturality arguments, a lower bound for this Majorana mass can be given.

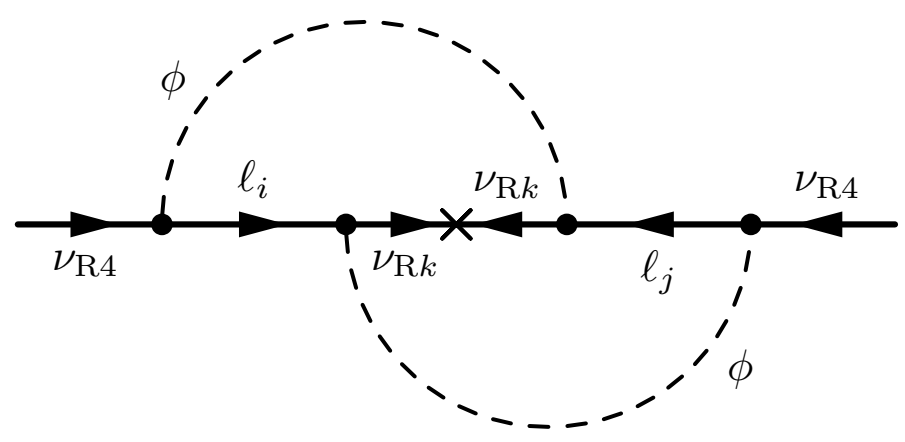

Figure 1: The two-loop process that provides a Majorana mass for the fourth-generation righthanded neutrino in the framework of type I see-saw. The indices $i$ and $j$ represent any of the four families; the index $k$, however, represents only the right-handed neutrinos associated to the generation of masses for the light families, $k=1,2,3$.

Let us consider first the case in which the three light neutrinos obtain their masses via a type-I see-saw containing heavy right-handed neutrinos with masses $m_{\mathrm{R} k}$ (with $k=1,2,3$ ) 
of the order of $10^{12}-10^{15} \mathrm{GeV}$. Since lepton number is not conserved it is natural to consider a Majorana mass term for the fourth right-handed neutrino, $\nu_{\mathrm{R} 4}$. However, in order to satisfy the LEP bounds on the number of light active neutrinos, $m_{\mathrm{R} 4}$ should be, at most, of the order of a few $\mathrm{TeV}$ and, therefore, much smaller than $m_{\mathrm{R} k}$. Thus, one might think that perhaps it is more natural to set directly $m_{\mathrm{R} 4}=0$ and consider only Dirac neutrinos for the fourth generation. The question that arises then is whether this choice is stable or not under radiative corrections and what is the natural size one might expect for $m_{\mathrm{R} 4}$, since setting $m_{\mathrm{R} 4}=0$ does not increase the symmetries of the Lagrangian. The answer can be obtained from the diagram in figure 1 which gives a logarithmically divergent contribution to $m_{\mathrm{R} 4}$ induced by the presence of the three heavy Majorana neutrino masses, $m_{\mathrm{R} k}[25,26]$. Thus, above the $m_{\mathrm{R} k}$ scale, $m_{\mathrm{R} 4}$ and $m_{\mathrm{R} k}$ mix under renormalization and do not run independently. Therefore, even if one finds a model in which $m_{\mathrm{R} 4}=0$ at some scale $\Lambda_{C}>m_{\mathrm{R} k}, m_{\mathrm{R} 4}$ will be generated by running from $\Lambda_{C}$ to $m_{\mathrm{R} k}$. This running can easily be estimated from the diagram in figure 1 and, barring accidental cancellations, one should require

$$
m_{\mathrm{R} 4} \gtrsim \frac{1}{(4 \pi)^{4}} \sum_{i j k} Y_{i 4} Y_{i k}^{*} m_{\mathrm{R} k} Y_{j k}^{*} Y_{j 4} \ln \left(\Lambda_{C} / m_{\mathrm{R} k}\right) \gtrsim \frac{1}{(4 \pi)^{4}} \sum_{i j k} Y_{i 4} Y_{i k}^{*} m_{\mathrm{R} k} Y_{j k}^{*} Y_{j 4}
$$

where $i, j=1,2,3,4, k=1,2,3$ and in the last step we have taken $\ln \left(\Lambda_{C} / m_{\mathrm{R} k}\right) \gtrsim 1$. Of course, given a particular renormalizable model yielding $m_{\mathrm{R} 4}=0$ at tree level (see appendix A for an explicit example) one should be able to compute the full two-loop mass $m_{\mathrm{R} 4}$, which will be finite and will contain the logarithmic contributions we have just discussed.

Eq. (3.1) sets the lower bound that we had announced. Let us now estimate its value; bearing in mind that in type I see-saw the light neutrino masses are given by ${ }^{3}$ $\left(m_{\nu}\right)_{i j} \sim \sum_{k} Y_{i k} Y_{j k} v_{\phi}^{2} / m_{\mathrm{R} k}$. Then, by taking all $m_{\mathrm{R} k}$ of the same order we can rewrite the bound as

$$
m_{\mathrm{R} 4} \gtrsim \sum_{i j} \frac{Y_{i 4}\left(m_{\nu}^{*}\right)_{i j} Y_{j 4}}{(4 \pi)^{4}} \frac{m_{\mathrm{R} k}^{2}}{v_{\phi}^{2}} .
$$

To give a conservative estimate we consider only the contribution of the first three generations because we expect their Yukawa couplings to the fourth right-handed neutrino to be somewhat suppressed due to universality and LFV constraints $[16,17,23,53]$ (say, $Y_{k 4} \sim 10^{-2}$ ). Once we fix the neutrino masses and the Yukawa couplings between the fourth-generation neutrino and the first three, $m_{\mathrm{R} 4}$ grows quadratically with $m_{\mathrm{R} k}$. For $m_{\nu}=0.01 \mathrm{eV}$ and $Y_{k 4}=0.01$ we obtain that $m_{\mathrm{R} 4}$ is of order keV, GeV, PeV for $m_{\mathrm{R} k}=10^{9}, 10^{12}, 10^{15} \mathrm{GeV}$, respectively. The contribution of the fourth active neutrino is not necessarily suppressed by the Yuwawa couplings and, in principle, by using it, even more restrictive bounds on $m_{\mathrm{R} 4}$ could be set. However, as $\left(m_{\nu}\right)_{44}$ is model-dependent ${ }^{4}$, we keep the most conservative bound.

\footnotetext{
${ }^{3}$ Notice that by integrating out the three heavy right-handed neutrinos we obtain a Majorana neutrino mass matrix for the four active neutrinos which is of the order of the light neutrino masses.

${ }^{4}$ In this case, $\left(m_{\nu}\right)_{44}$ is the see-saw mass induced by only the three heavy right-handed neutrinos, thus,
} 


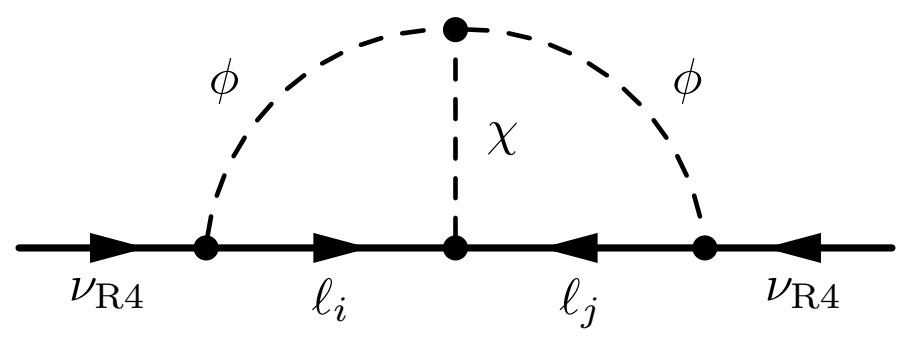

Figure 2: The process that provides a Majorana mass for the right-handed neutrino associated to the fourth generation in the framework of type II see-saw.

Let us consider now the case in which the three light neutrinos obtain their masses through the type II see-saw mechanism (see section 2.2.2), i.e., through their coupling to a scalar triplet, $\chi$, which develops a VEV. As discussed in section 3, this triplet cannot be the only source Majorana masses for the fourth-generation neutrinos and, at least, one right-handed neutrino is needed. We will assume then that there is a right-handed neutrino which has Yukawa couplings to the four SM doublets. In this scenario one can easily see that the right-handed neutrino will acquire, at two loops (as seen in figure 2), a Majorana mass. This just reflects the fact that the right-handed neutrino mass $m_{\mathrm{R} 4}$ and the trilinear coupling of the triplet, $\mu$, mix under renormalization. Applying the same arguments used in the case of see-saw type I for light neutrino masses and the estimate of the diagram in figure 2 we can write

$$
m_{\mathrm{R} 4} \gtrsim \frac{\mu}{(4 \pi)^{4}} \sum_{i j} Y_{i 4}\left(Y_{\chi}^{*}\right)_{i j} Y_{j 4},
$$

where $Y_{\chi}$ are the Yukawa couplings of the triplet to the lepton doublets and, as before, we have taken $\ln \left(\Lambda_{C} / m_{\chi}\right) \gtrsim 1$. As in the type I see-saw case the result can also be expressed in terms of the light neutrino masses $\left(m_{\nu}\right)_{i j} \sim\left(Y_{\chi}\right)_{i j} \mu v_{\phi}^{2} / m_{\chi}^{2}$; thus

$$
m_{\mathrm{R} 4} \gtrsim \sum_{i j} \frac{Y_{i 4}\left(m_{\nu}^{*}\right)_{i j} Y_{j 4}}{(4 \pi)^{4}} \frac{m_{\chi}^{2}}{v_{\phi}^{2}}
$$

which shows a similar structure to that obtained for type I see-saw, eq. (3.2). The same result is obtained for type III see-saw, whose couplings are analogous to those of type I.

The similarity of the two results suggests that bounds of this type are quite general and should appear in all kinds of four-generation models with light Majorana neutrinos. In fact, as discussed in section 2.4, light Majorana neutrino masses can be parametrized in many models by means of the Weinberg operator, eq. (2.13), which yields neutrino masses given by eq. (2.14). Then, one could draw a two-loop diagram analogous to the diagrams in figures 1 and 2 but with the propagators of heavy particles pinched and substituted by one insertion of the Weinberg operator. This diagram is quadratically divergent and, therefore, its contribution to $m_{\mathrm{R} 4}$ can not be reliably computed in the effective field theory because it depends on the details of the matching with the full theory from which the

it could even be zero if the Yukawas between the fourth lepton doublet and the three right-handed neutrinos vanish for some reason. 
effective one originates (in fact it vanishes in dimensional regularization or in any other regularization scheme allowing symmetric integration), but one can use naive dimensional analysis to estimate contributions of order

$$
m_{\mathrm{R} 4} \sim \frac{\Lambda_{W}}{(4 \pi)^{4}} \sum_{i j} Y_{i 4} c_{i j}^{*} Y_{j 4} \sim \frac{Y_{i 4}\left(m_{\nu}^{*}\right)_{i j} Y_{j 4}}{(4 \pi)^{4}} \frac{\Lambda_{W}^{2}}{v_{\phi}^{2}},
$$

which is precisely the result obtained in the see-saw models discussed above if one identifies $\Lambda_{W} \sim m_{\mathrm{R} k}, m_{\chi}$. However, it is important to remark that in the low energy effective theory $m_{\mathrm{R} 4}$ is a free parameter, and eq. (3.5) is only a naive dimensional analysis estimate of what one would expect in a more complete theory.

\section{Light neutrino masses induced by new generations}

After the discussion above, to describe correctly the neutrino sector of models with four generations we need just one relatively light right-handed neutrino, $\nu_{\mathrm{R}}$, to give Dirac mass terms to the fourth-generation neutrinos, while Majorana masses for light neutrinos can be parametrized by the Weinberg operator. We will work in this minimal four-generation scenario, thus, the relevant part of the Lagrangian for our discussion is

$$
\mathcal{L}_{Y}=-\bar{\ell} Y_{e} e_{\mathrm{R}} \phi-\bar{\ell} y \nu_{\mathrm{R}} \tilde{\phi}-\frac{1}{2} \overline{\nu_{\mathrm{R}}^{\mathrm{c}}} m_{\mathrm{R}} \nu_{\mathrm{R}}+\frac{1}{2 v_{\phi}^{2}}(\bar{\ell} \tilde{\phi}) m_{\mathrm{L}}\left(\phi^{\dagger} \tilde{\ell}\right)+\text { H.c. }
$$

where $\ell$ and $e_{\mathrm{R}}$ contain the four generation components while $\nu_{\mathrm{R}}$ is the only right-handed neutrino. Thus $Y_{e}$ is a completely general $4 \times 4$ complex matrix, $y$ is a 4 component column vector, $m_{\mathrm{R}}$ is just a number and $m_{\mathrm{L}}$ is a general complex symmetric $4 \times 4$ matrix. The Dirac limit is recovered when $m_{\mathrm{R}}=0$ and $m_{\mathrm{L}}=0$. Since light neutrino masses are very small, we will assume $m_{\mathrm{L}} \ll v_{\phi}$ while $m_{\mathrm{R}}$, as we immediately see, cannot be very large to ensure there are only three light active neutrinos. Moreover, as shown in the previous section, we do not expect it to be zero if $m_{\mathrm{L}}$ is not zero.

Above we have taken for $m_{\mathrm{L}}$ a general complex symmetric $4 \times 4$ matrix in spite of the fact that to describe the light neutrino sector we just need a $3 \times 3$ matrix. This is because in most of the neutrino mass models one also obtains contributions to the fourthgeneration Weinberg operator. For instance, we give below the values of $m_{\mathrm{L}}$ one obtains for the different types of seesaw.

If the three light neutrino masses are generated by the seesaw mechanism type I or type III, we need three of the right-handed neutrinos much heavier than the fourth. We can always choose a basis in which the Majorana mass matrix of right-handed neutrinos is diagonal and integrate out the three heavy right-handed neutrinos. The result can be writen in terms of the Weinberg operator in (4.1) with

$$
\left(m_{\mathrm{L}}\right)_{\alpha \beta}=-\sum_{k=1,2,3} \frac{\left(Y_{\nu}\right)_{\alpha k}\left(Y_{\nu}\right)_{\beta k}}{m_{\mathrm{R} k}} v_{\phi}^{2},
$$


where $m_{\mathrm{R} k}$ are the eigenvalues of the diagonal Majorana mass matrix of the three heavy right-handed neutrinos, while $\left(Y_{\nu}\right)_{\alpha k}$ are the Yukawa couplings of the three heavy righthanded neutrinos with the four lepton doublets. Then, the $4 \times 4$ mass matrix (4.2) is projective and has at most rank 3 .

If the three light neutrino masses are generated by the VEV of a triplet (type II see-saw), we will have

$$
\left(m_{\mathrm{L}}\right)_{\alpha \beta}=2\left(Y_{\chi}\right)_{\alpha \beta} v_{\chi},
$$

being $\left(Y_{\chi}\right)_{\alpha \beta}$ the Yukawa couplings of the 4 lepton doublets to the triplet and $v_{\chi} \sim \mu v_{\phi}^{2} / m_{\chi}^{2}$ its VEV. In this case, $m_{\mathrm{L}}$ is a completely general $4 \times 4$ symmetric complex matrix.

After SSB the neutrino mass matrix (in the basis $\left(\nu_{\mathrm{L} \alpha}^{\mathrm{c}}, \nu_{\mathrm{R}}\right)$ ) is

$$
M=\left(\begin{array}{cc}
m_{\mathrm{L}} & y v_{\phi} \\
y^{\mathrm{T}} v_{\phi} & m_{\mathrm{R}}
\end{array}\right) .
$$

To diagonalize this mass matrix we perform first a $4 \times 4$ rotation in order to separate heavy from light degrees of freedom, so we change from the flavour basis $\left(\nu_{e}, \nu_{\mu}, \nu_{\tau}, \nu_{E}\right)$ to a new basis $\nu_{1}^{\prime}, \nu_{2}^{\prime}, \nu_{3}^{\prime}, \nu_{4}^{\prime}$ in which the first three states are light (with masses given by $m_{\mathrm{L}}$ ) and only $\nu_{4}^{\prime}$ mixes with $\nu_{\mathrm{R}}$. Then, we have $\nu_{\alpha}=\sum_{i} V_{\alpha i} \nu_{i}^{\prime}(i=1, \cdots, 4, \alpha=e, \mu, \tau, E)$, where $V$ is a orthogonal matrix, and we define

$$
N_{\alpha} \equiv V_{\alpha 4}=\frac{y_{\alpha}}{\sqrt{\sum_{\beta} y_{\beta}^{2}}} .
$$

Now, we are free to choose $\nu_{1}^{\prime}, \nu_{2}^{\prime}, \nu_{3}^{\prime}$ in any combination of $\nu_{e}, \nu_{\mu}, \nu_{\tau}, \nu_{E}$ as long as they are orthogonal to $\nu_{4}^{\prime}$, i.e., $\sum_{\alpha} V_{\alpha k} N_{\alpha}=0$ for $k=1,2,3$. The orthogonality of $V$ almost fixes all its elements in terms of $N_{\alpha}$, but still leaves us some freedom to set three of them to zero. Following $[16,17]$ we choose $V_{\tau 1}=V_{E 1}=V_{E 2}=0$ for convenience. The transpose of the matrix $V$ is:

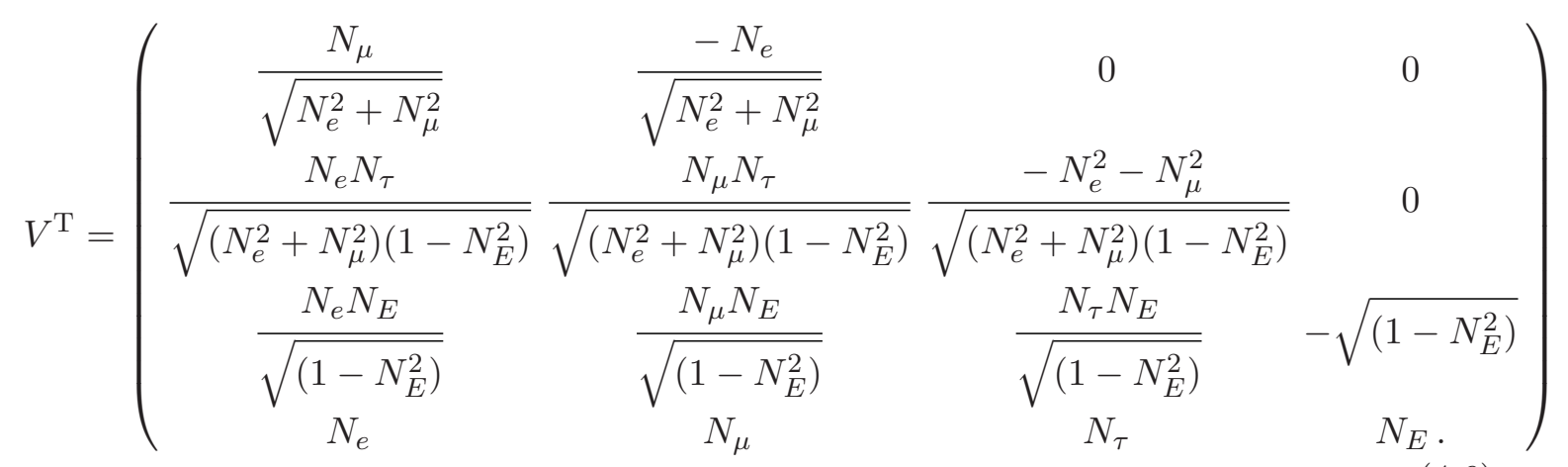

After this rotation the neutrino mass matrix is

$$
\tilde{M}=\left(\begin{array}{ccccc} 
& & & \omega_{1} & 0 \\
\tilde{m}_{\mathrm{L}} & & & \omega_{2} & 0 \\
& & & \omega_{3} & 0 \\
\omega_{1} & \omega_{2} & \omega_{3} & \omega_{4} & m_{\mathrm{D}} \\
0 & 0 & 0 & m_{\mathrm{D}} & m_{\mathrm{R}}
\end{array}\right),
$$


where $\left(\tilde{m}_{\mathrm{L}}\right)_{k k^{\prime}}=\left(V m_{\mathrm{L}} V^{\mathrm{T}}\right)_{k k^{\prime}}$ is a $3 \times 3$ matrix with $k, k^{\prime}=1,2,3, \omega_{k}=\left(V m_{\mathrm{L}} V^{\mathrm{T}}\right)_{4 k}$, $\omega_{4}=\left(V m_{\mathrm{L}} V^{\mathrm{T}}\right)_{44}$ and $m_{\mathrm{D}}=v_{\phi} \sqrt{\sum_{\alpha} y_{\alpha}^{2}}$. Since $\tilde{m}_{\mathrm{L}}, \omega_{k}, \omega_{4} \ll m_{\mathrm{R}}, m_{\mathrm{D}}$, the matrix $\tilde{M}$ can be block-diagonalized using the see-saw formula. Then, the mass matrix of the light neutrinos (at tree level) will be

$$
m_{\nu}^{(0)}=\tilde{m}_{\mathrm{L}}-\frac{m_{\mathrm{R}}}{m_{\mathrm{R}} \omega_{4}-m_{\mathrm{D}}^{2}} \vec{\omega} \cdot \vec{\omega}^{\mathrm{T}},
$$

while the heavy sector will be obtained after diagonalizing the $2 \times 2$ matrix

$$
M_{H}=\left(\begin{array}{cc}
\omega_{4} & m_{\mathrm{D}} \\
m_{\mathrm{D}} & m_{\mathrm{R}}
\end{array}\right) .
$$

Neglecting $\omega_{4}$, this diagonalization leads to two Majorana neutrinos

$$
\begin{aligned}
& \nu_{4}=i \cos \theta\left(-\nu_{4}^{\prime}+\nu_{4}^{\prime \mathrm{c}}\right)+i \sin \theta\left(\nu_{\mathrm{R}}-\nu_{\mathrm{R}}^{\mathrm{c}}\right) \\
& \nu_{\overline{4}}=-\sin \theta\left(\nu_{4}^{\prime}+\nu_{4}^{\prime \mathrm{c}}\right)+\cos \theta\left(\nu_{\mathrm{R}}+\nu_{\mathrm{R}}^{\mathrm{c}}\right)
\end{aligned}
$$

with masses

$$
m_{4, \overline{4}}=\frac{1}{2}\left(\sqrt{m_{\mathrm{R}}^{2}+4 m_{\mathrm{D}}^{2}} \mp m_{\mathrm{R}}\right),
$$

and mixing angle $\tan ^{2} \theta=m_{4} / m_{\overline{4}}$. The imaginary unit factor $i$ and the relative signs in $\nu_{4}$ are necessary to keep the mass terms positive and preserve the canonical Majorana condition $\nu_{4}=\nu_{4}^{\mathrm{c}}$. If $m_{\mathrm{R}} \ll m_{\mathrm{D}}$, we have $m_{4} \approx m_{\overline{4}}, \tan \theta \approx 1$, and we say we are in the pseudo-Dirac limit while when $m_{\mathrm{R}} \gg m_{\mathrm{D}}, m_{4} \approx m_{\mathrm{D}}^{2} / m_{\mathrm{R}}$ and $m_{\overline{4}} \approx m_{\mathrm{R}}, \tan \theta \approx m_{\mathrm{D}} / m_{\mathrm{R}}$ and we say we are in the see-saw limit.

Eq. (4.8) can be used as long as $m_{\mathrm{R}} \omega_{4}-m_{\mathrm{D}}^{2}$ is different from zero. However, we expect $m_{\mathrm{R}}$ to be below few $\mathrm{TeV}$ and $\omega_{4}$ below $1 \mathrm{eV}$. Therefore $m_{\mathrm{R}} \omega_{4} \ll m_{\mathrm{D}}^{2}$ unless $m_{\mathrm{D}}$ is very small but, in that case, the fourth-generation neutrinos will be too light. Thus, the correction to the $3 \times 3$ neutrino mass matrix is projective (only one eigenvalue different from zero) and it is naturally order $m_{\mathrm{L}}^{2}$ and, therefore, negligible.

Summarizing, there are two heavy neutrinos 4 and $\overline{4}$ (with a small pollution from $m_{\mathrm{L}}$ which can be neglected) and a tree-level mass matrix for the light neutrinos $m_{\nu}^{(0)} \simeq \tilde{m}_{\mathrm{L}}$. Therefore, neglecting the small $\omega_{i}$ 's in eq. (4.7), the $5 \times 5$ unitary matrix which relates the flavour with the mass eigenstate basis can be written as $U=U_{H} \cdot U_{L}$, being $U_{H}$ the rotation in the heavy sector which diagonalizes the mass matrix $M_{H}$ in eq. (4.9) and $U_{L}$ given by

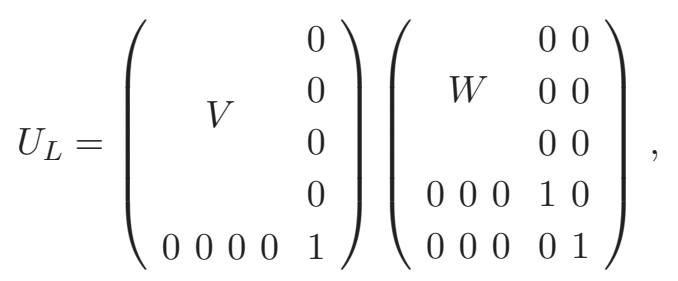

where $V$ rotates from the $\nu_{i}^{\prime}$ basis to the flavour basis (see eq. (4.6)) and $W$ is the matrix which diagonalizes $\tilde{m}_{\mathrm{L}}$. Within this approximation, the mixing among the light and the heavy sector, which we wish to constrain, depends on $\left(U_{L}\right)_{\alpha 4}=V_{\alpha 4}=N_{\alpha}$. 
Having fixed the tree-level neutrino mass spectrum and given the huge hierarchies present we should consider the stability of the results against radiative corrections. One can check that there are no rank-changing one-loop corrections to the neutrino mass matrices. This result can be easily understood in the $\nu_{i}^{\prime}$ basis that we defined before, since the light neutrinos $\left(\nu_{1}^{\prime}, \nu_{2}^{\prime}, \nu_{3}^{\prime}\right)$ are decoupled from the heavy sector, $\nu_{4}, \nu_{\overline{4}}$, so there are not one-loop diagrams involving the fourth-generation neutrinos with light ones as external legs.

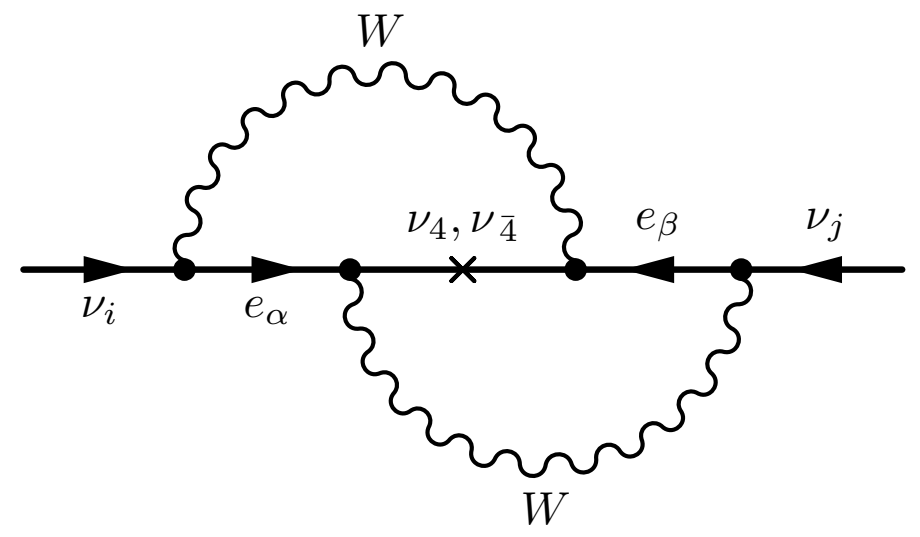

Figure 3: Two-loop diagram generating light neutrino masses in the presence of a Majorana fourth generation.

However it has been shown $[16,17,23,27]$ that two-loop corrections induced by the fourth-generation fermions can generate neutrino masses for the light neutrinos even if they were not present at tree level, see figure 3. In the $\nu_{i}^{\prime}$ basis the result reads (see [23] for details)

$$
\left(m_{\nu}\right)_{i j}^{(2)}=-\frac{g^{4}}{m_{W}^{4}} m_{\mathrm{R}} m_{\mathrm{D}}^{2} \sum_{\alpha} V_{\alpha i} V_{\alpha 4} m_{\alpha}^{2} \sum_{\beta} V_{\beta j} V_{\beta 4} m_{\beta}^{2} I_{\alpha \beta},
$$

where the sums run over the charged leptons $\alpha, \beta=e, \mu, \tau, E$ while $i, j=1,2,3$, and $I_{\alpha \beta}$ is a loop integral which was discussed in [23]. When $m_{\mathrm{R}}=0,\left(m_{\nu}\right)_{i j}^{(2)}=0$, as it should, because in that case lepton number is conserved. Also when $m_{\mathrm{D}}=0$ we obtain $\left(m_{\nu}\right)_{i j}^{(2)}=0$, since then the right-handed neutrino decouples completely and lepton number is again conserved.

To see more clearly the structure of this mass matrix we can approximate $m_{e}=$ $m_{\mu}=m_{\tau}=0$; then, since we have chosen $V_{\tau 1}=V_{E 1}=V_{E 2}=0$, the only non-vanishing element in $\left(m_{\nu}\right)_{i j}^{(2)}$ is $\left(m_{\nu}\right)_{33}^{(2)}$ and it is proportional to $V_{E 3}^{2} N_{E}^{2} m_{E}^{4} I_{E E}$. Therefore, the largest contribution to $\left(m_{\nu}\right)^{(2)}$ is given by:

$$
\begin{aligned}
\left(m_{\nu}\right)_{33}^{(2)} & =-\frac{g^{4}}{m_{W}^{4}} N_{E}^{2}\left(N_{e}^{2}+N_{\mu}^{2}+N_{\tau}^{2}\right) m_{\mathrm{R}} m_{\mathrm{D}}^{2} m_{E}^{4} I_{E E} \\
& \approx \frac{g^{4}}{2(4 \pi)^{4}}\left(N_{e}^{2}+N_{\mu}^{2}+N_{\tau}^{2}\right) m_{\mathrm{R}} \frac{m_{\mathrm{D}}^{2} m_{E}^{2}}{m_{W}^{4}} \ln \frac{m_{E}}{m_{\overline{4}}}
\end{aligned}
$$

where in the last line we have used the approximated expression of the loop integral $I_{E E}$ in the case $m_{E} \gg m_{4, \overline{4}} \gg m_{W}$ for definiteness, but other mass relations lead to analogous conclusions. Keeping all the charged lepton masses one can easily show that the eigenvalues 
of the light neutrino mass matrix are proportional to $m_{\mu}^{4}, m_{\tau}^{4}, m_{E}^{4}$ which gives a huge hierarchy between neutrino masses. Therefore, as discussed in $[23,26]$, these radiative corrections cannot explain by themselves the observed spectrum of masses and mixings, although they lead to a strong constraint for this kind of SM extensions which we will analyze in the next section.

\section{Phenomenological constraints}

\subsection{Direct searches}

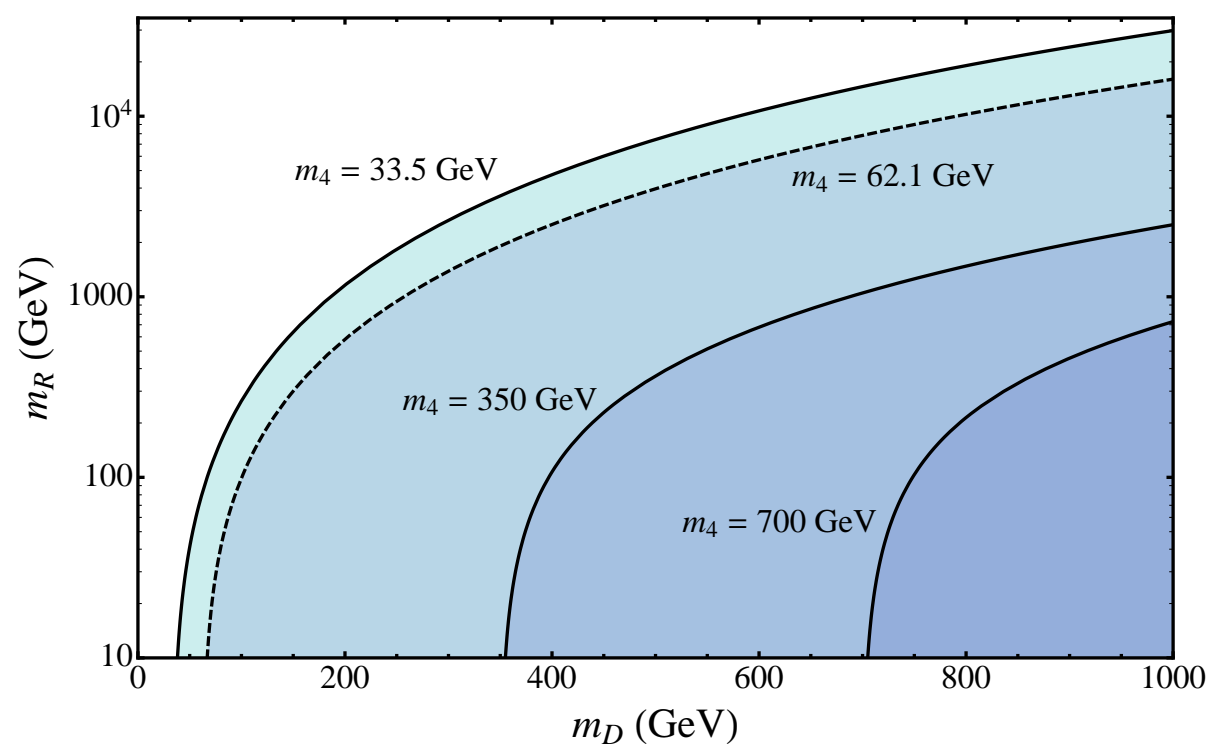

Figure 4: The shaded region shows the allowed values for the Majorana and Dirac masses of the heavy neutrinos given the LEP bound $m_{N}>33.5 \mathrm{GeV}$ on stable neutrinos with both Dirac and Majorana masses. We also display a dashed line in the $62.1 \mathrm{GeV}$ limit for unstable neutrinos. Two more lines are drawn for completeness, giving an idea of the combination of parameters that produces two possible, allowed masses for the lightest heavy neutrino.

Let us now discuss the constraints that several phenomenological tests impose on the parameters of this minimal four-generation $(4 \mathrm{G})$ model. Direct searches for the new heavy leptons can be used to set limits on the Yukawa couplings and the Majorana mass of the $\nu_{\mathrm{R}}$. In the case of the heavy charged lepton, searches at LEP [4] yield $m_{E}>100.8 \mathrm{GeV}$ (assuming it decays rapidly to $\nu W$; a slightly poorer bound is obtained if the lepton is long-lived and can be tracked inside the detectors), which can be immediately translated into a bound on the corresponding Yukawa. For the heavy neutrinos, we can have different bounds depending on their stability and the Dirac or Majorana character of their masses [4]: stable neutrinos, for example (understood here as 'stable enough to get out of the detectors after production'), are only constrained by the requirement that they don't show up in the invisible decays of the $Z$ boson. Unstable (visible) neutrinos get tighter bounds due to the non-observation of their decay products. As we are not making any a priori assumption about the neutrino mass structure, we will select here the most conservative from this set of 
bounds; that corresponds to a stable neutrino with both Dirac and Majorana mass terms, for which we demand $m_{N}>33.5 \mathrm{GeV}$ [54]. The weakest bound for an unstable neutrino, which applies if it has again both Dirac and Majorana mass terms, will also be of use; we need in that case $m_{N}>62.1 \mathrm{GeV}$ [20]. As these bounds apply to the physical masses of the neutrinos, which as seen in eqs. (4.10-4.12) are nonlinear combinations of the Dirac and Majorana components, we display in figure 4 the translation of the 33.5 and $62.1 \mathrm{GeV}$ bounds into the $m_{\mathrm{D}}-m_{\mathrm{R}}$ plane, together with several other lines to give an idea of the relations between physical masses and Lagrangian parameters.

As explained in section 4, the neutrino Yukawas $y_{\alpha}$ encode the mixings between the flavour-eigenstate neutrinos $\nu_{\alpha}$ and the mass eigenstates $\nu_{4, \overline{4}}$. Thus, we can use mixingmediated LFV processes to constrain the values of the light neutrino Yukawas $y_{e}, y_{\mu}, y_{\tau}$. It is important to note, however, that the situation is not the same for 'stable' and unstable neutrinos; so-called stable neutral leptons are constrained to decay outside the detectors, which implies that the mean free path must go beyond $\mathcal{O}(\mathrm{m})$. The lightest of our heavy neutrinos can only decay through mixing (the main channel being $\nu_{4} \rightarrow \ell_{\alpha} W, \alpha=e, \mu, \tau$, with a possibly virtual $W$ depending on the mass of the $\nu_{4}$ ), so this statement is actually a constraint on the Yukawas, implying $y_{\alpha} \sim N_{\alpha} \lesssim 10^{-6}$. This constraint is much stronger than any other phenomenolgical bound, and so it ends the discussion for stable neutrinos, which must have very small mixings that won't be observable in low-energy experiments in the near future (see below). For the rest of this section we will consider the case of unstable neutrinos, which present a richer variety of constraints.

\subsection{Lepton flavour violation}

Let us now discuss the bounds on violation of lepton family number that can shed light on the relevant mixings of our model; the most stringent limits are derived from the nonobservation of radiative decays of the form $\ell_{\alpha} \rightarrow \ell_{\beta} \gamma^{5}$. In our model, the ratios for such processes are given by

$$
B\left(\ell_{\alpha} \rightarrow \ell_{\beta} \gamma\right) \equiv \frac{\Gamma\left(\ell_{\alpha} \rightarrow \ell_{\beta} \gamma\right)}{\Gamma\left(\ell_{\alpha} \rightarrow \ell_{\beta} \nu \bar{\nu}\right)}=\frac{3 \alpha}{2 \pi}\left|\sum_{a=4, \overline{4}} U_{\beta a} U_{\alpha a}^{*} H\left(m_{a}^{2} / m_{W}^{2}\right)\right|^{2},
$$

where $H(x)$ is a loop function that can be found in [53], and the sum proceeds over all the heavy neutrinos ${ }^{6}$ (one in the Dirac case, two if they are Majorana). The weakest bounds are obtained if only one neutrino with light mass runs inside the loop; this corresponds either to the Dirac limit with a low mass or to a hard see-saw limit, with the heavy neutrino almost decoupled due to its small mixing. We will assume this scenario in our calculations in order to produce conservative bounds. Table 1 summarises the experimental limits and the constraints that can be extracted from these processes.

\footnotetext{
${ }^{5}$ Bounds obtained from present data on $\mu-e$ conversion in nuclei [4] are of the same order. However, there are plans to improve the sensitivity in $\mu-e$ conversion in 4 and even 6 orders of magnitude [55], therefore we expect from this process much stronger bounds in the future.

${ }^{6}$ Note this expression contains the contributions from the light neutrinos; by using unitarity of the mixing matrix, they are included in the definition of $H(x)$.
} 


\begin{tabular}{|c|c|}
\hline Experimental bounds at 90\% C.L. $[4,56]$ & Constraints on the mixings \\
\hline$B(\mu \rightarrow e \gamma)<2.4 \times 10^{-12}$ & $N_{e} N_{\mu}<2.85 \times 10^{-4}$ \\
\hline$B(\tau \rightarrow e \gamma)<1.85 \times 10^{-7}$ & $N_{e} N_{\tau}<0.079$ \\
\hline$B(\tau \rightarrow \mu \gamma)<2.5 \times 10^{-7}$ & $N_{\mu} N_{\tau}<0.093$ \\
\hline
\end{tabular}

Table 1: Summary of the constraints derived from low-energy radiative decays.

\subsection{Universality tests}

A second class of constraints upon family mixing arises from the tests of universality in weak interactions. For our purposes, these are either direct comparison of decay rates of one particle into two different weak-mediated channels, or comparison of the decay rates of two different particles into the same channel ${ }^{7}$. If the weak couplings are to be the same for all families these rates should differ only in known kinematic factors or calculable higher-order corrections. The relevant ratios are:

$$
\begin{array}{rlrl}
R_{\pi \rightarrow e / \pi \rightarrow \mu} & \equiv \frac{\Gamma(\pi \rightarrow e \nu)}{\Gamma(\pi \rightarrow \mu \nu)}, & R_{\tau \rightarrow \mu / \tau \rightarrow e} \equiv \frac{\Gamma(\tau \rightarrow \mu \nu \bar{\nu})}{\Gamma(\tau \rightarrow e \nu \bar{\nu})}, \\
R_{\tau \rightarrow e / \mu \rightarrow e} \equiv \frac{\Gamma(\tau \rightarrow e \nu \bar{\nu})}{\Gamma(\mu \rightarrow e \nu \bar{\nu})}, & R_{\tau \rightarrow \mu / \mu \rightarrow e} \equiv \frac{\Gamma(\tau \rightarrow \mu \nu \bar{\nu})}{\Gamma(\mu \rightarrow e \nu \bar{\nu})},
\end{array}
$$

and their theoretical values in a $3 \mathrm{G} \mathrm{SM}$ can be consulted, for example, in [58]. Comparison of the experimental values and the $3 \mathrm{G}$ predictions yields values very close to 1 , as can be seen in table 2; in our 4G model, family mixing induces deviations from this behaviour that must be kept under control. Essentially, these deviations result from the fact that the flavour-eigenstate neutrinos $\nu_{e}, \nu_{\mu}, \nu_{\tau}$ have a small component of the heavy neutrinos $\nu_{4}, \nu_{\overline{4}}$, which cannot be produced in the decays of pions, taus or muons; the corresponding mixings $N_{e}, N_{\mu}, N_{\tau}$ are then forced to be small. In table 2 we also show the constraints that this processes impose on the mixing parameters.

\begin{tabular}{|l|l|}
\hline Experimental bounds at 90\% C.L. [4] & Constraints on the mixings \\
\hline$\frac{R_{\pi \rightarrow e / \pi \rightarrow \mu}}{R_{\pi \rightarrow e / \pi \rightarrow \mu}^{\mathrm{SM}}}=0.996 \pm 0.005$ & $N_{e}^{2}-N_{\mu}^{2}=0.004 \pm 0.005$ \\
\hline$\frac{R_{\tau \rightarrow e / \tau \rightarrow \mu}}{R_{\tau \rightarrow e / \tau \rightarrow \mu}^{\mathrm{SM}}}=1.000 \pm 0.007$ & $N_{e}^{2}-N_{\mu}^{2}=0.000 \pm 0.007$ \\
\hline$\frac{R_{\tau \rightarrow e / \mu \rightarrow e}}{R_{\tau \rightarrow e / \mu \rightarrow e}^{\mathrm{SM}}}=1.003 \pm 0.007$ & $N_{\mu}^{2}-N_{\tau}^{2}=0.003 \pm 0.007$ \\
\hline$\frac{R_{\tau \rightarrow \mu / \mu \rightarrow e}}{R_{\tau \rightarrow \mu / \mu \rightarrow e}^{\mathrm{SM}}}=1.001 \pm 0.007$ & $N_{e}^{2}-N_{\tau}^{2}=0.001 \pm 0.007$ \\
\hline
\end{tabular}

Table 2: Summary of the constraints derived from universality tests in weak decays. The ratios marked as "SM" represent the theoretical predictions of a 3G Standard Model.

\footnotetext{
${ }^{7}$ Data from neutrino oscillations can also be used to constrain the elements of the leptonic mixing matrix [57], however, they lead to weaker bounds than the ones obtained here.
} 

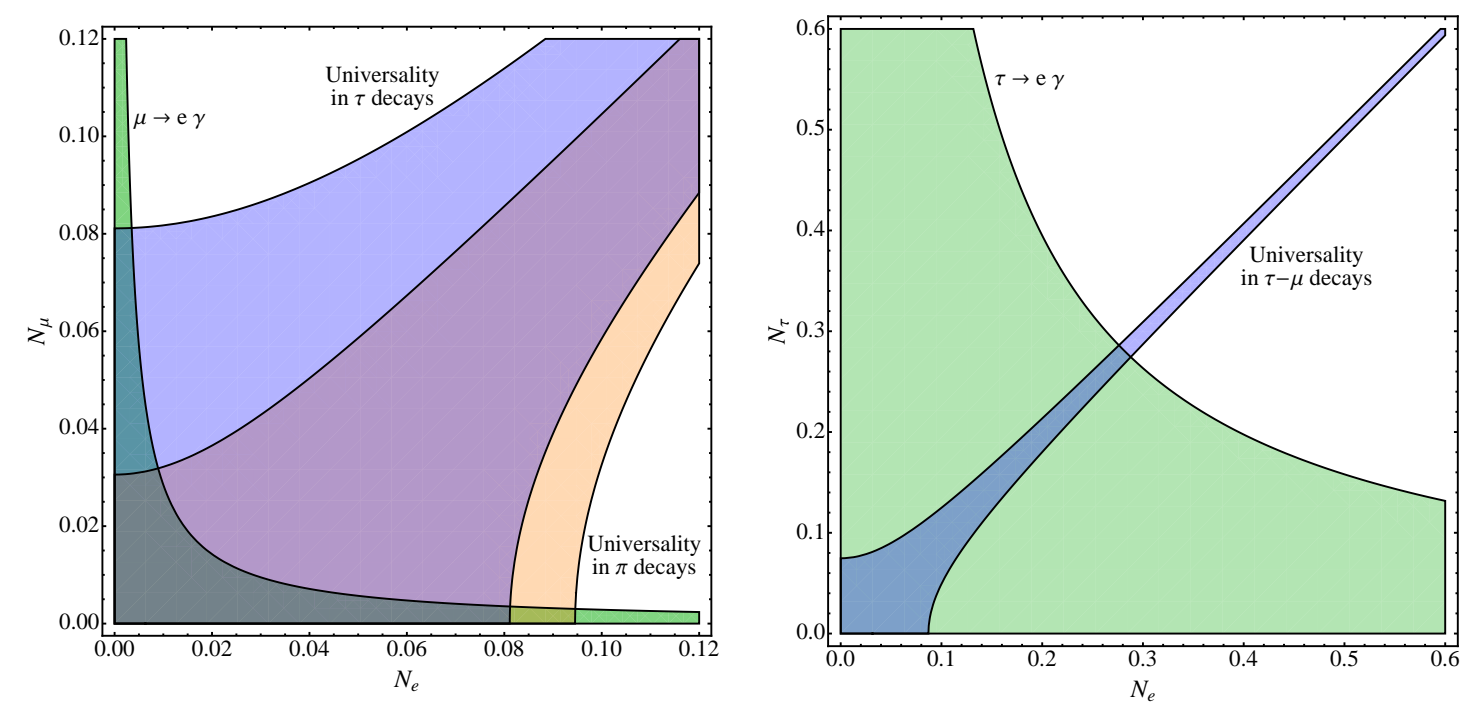

Figure 5: These two graphs present the allowed regions for the mixing parameters in our model at $90 \%$ confidence level, according to several LFV tests. The left plot displays the constraints in the $N_{e}-N_{\mu}$ plane, which are much more stringent and suffice to bound both $N_{e}$ and $N_{\mu}$. The right plot displays the $N_{e}-N_{\tau}$ plane; the $N_{\mu}-N_{\tau}$ plane offers slighly poorer constraints and is not displayed.

In figure 5 we collect all the relevant LFV constraints from tables 1 and 2. As can be read from the graphs, the final bounds we can set on the mixings of the light families are

$$
\begin{aligned}
& N_{e}<0.08 \\
& N_{\mu}<0.03 \\
& N_{\tau}<0.3
\end{aligned}
$$

\subsection{Light neutrino masses}

Finally, there is a further constraint that can be set upon the mixings of the model: as explained in section 4, the two-loop mechanism which gives small Majorana masses for the light neutrinos cannot explain by itself the observed pattern of masses in this simple model; it, nevertheless, still has the potential to generate too large masses, which would exclude the model. Of course, one could always invoke cancellations between these twoloop masses and other contributions (for example, the Weinberg operator), but we think that this wouldn't be a natural situation and choose not to consider it. If we bar such cancellations we need to impose that the two-loop masses don't go above some limit, and thus a bound can be set upon the parameters that participate in the two-loop mechanism, essentially the mixings and the Majorana mass, as seen in equation (4.14). Figure 6 shows the allowed regions for this constraint; the curves are constructed using the lowest possible values of the fourth-generation Dirac masses, in order to provide conservative limits (this 


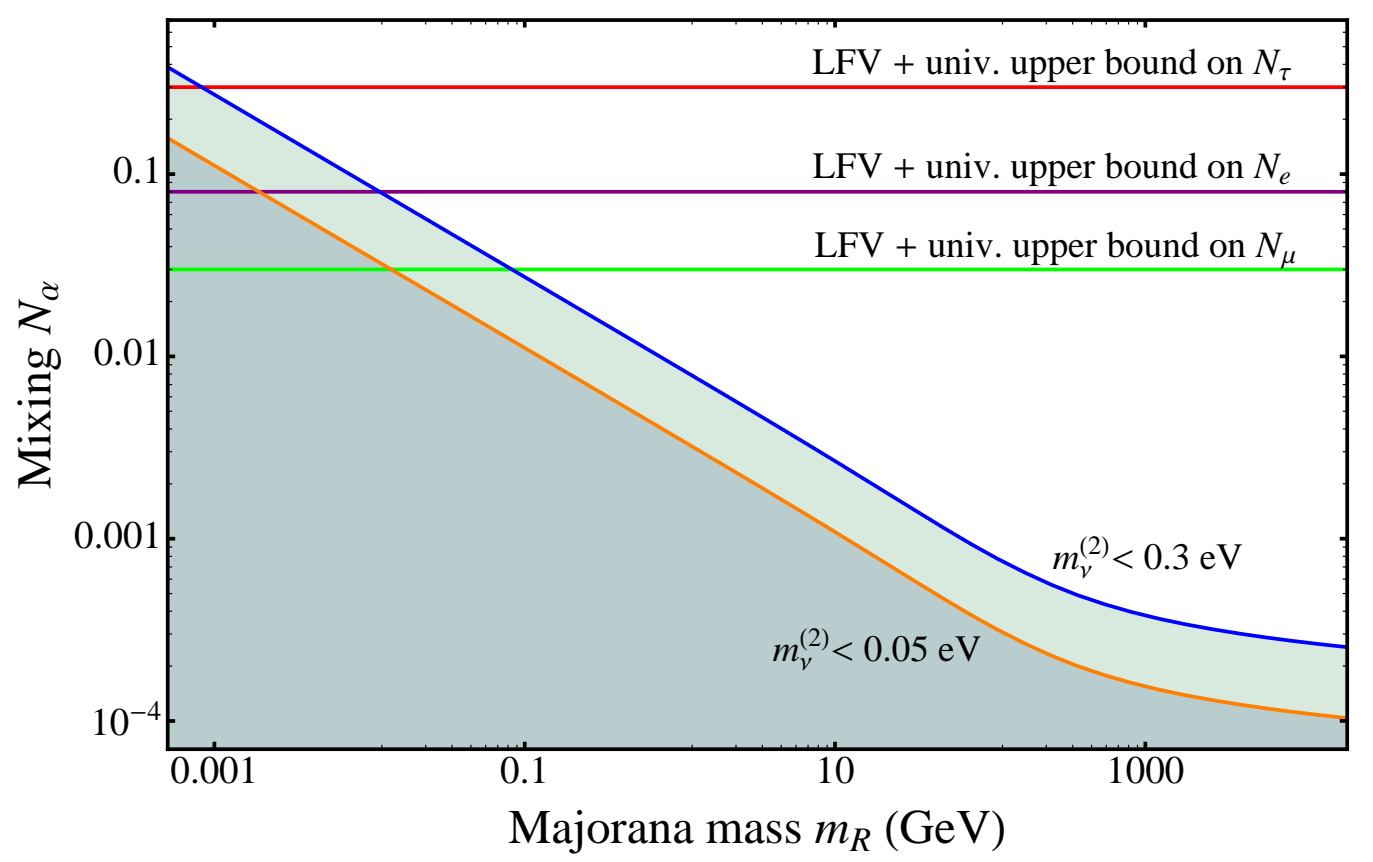

Figure 6: Summary of the constraints on the mixings of the model, as defined in equation (4.5). The three horizontal lines present the upper bounds in equation (5.2), derived from universality tests and limits on LFV processes. The two shaded areas display the allowed region derived from the fact that the two-loop diagram does not disturb the correct structure for the light neutrino masses, assumed to arise from any other mechanism. This last bound applies to any of the mixings.

implies using a different value of $m_{\mathrm{D}}$ for each $m_{\mathrm{R}}$, as we must also impose that $m_{4}$ is above $62.1 \mathrm{GeV}$ ). We show two possible limits: $m_{\nu}^{(2)}<0.05 \mathrm{eV}$ ensures that the largest two-loop mass is below the atmospheric mass scale; this, of course, does not guarantee that it doesn't distort the neutrino spectrum, which may contain smaller masses, so this bound can be contemplated as rather conservative (particular models may need to impose a stronger bound to be phenomenologically viable). An even more conservative constraint is obtained if we impose $m_{\nu}^{(2)}<0.3 \mathrm{eV}$, meaning that the largest of the two-loop masses is not above the bound imposed by cosmology to each mass of the degenerate spectrum, $\sum_{k} m_{k} \lesssim 1 \mathrm{eV}[59,60]$. Two-loop masses as large as $0.3 \mathrm{eV}$ will in most cases spoil the structure of neutrino masses, but there may be pathological cases in which such situation is allowed (for example, if the Weinberg operator generates a massless neutrino and two massive ones near the $0.3 \mathrm{eV}$ limit; then the two-loop diagram might provide the third mass to fit the mass splittings). Even with these conservative assumptions, the two-loop bound proves to be much stronger than those derived from universality and LFV for most of the parameter space. It is, therefore, a limit to be kept in mind when considering $4 \mathrm{G}$ models with Majorana neutrinos.

\subsection{Neutrinoless double beta decay $(0 \nu 2 \beta)$}

In our framework, the contributions to the amplitude of neutrinoless double beta decay 
$(0 \nu 2 \beta)$ can be written as:

$$
A=A_{L}+A_{\mathrm{md}}+A_{4},
$$

where $A_{L}$ stands for the light neutrino contribution (i.e., neutrino masses $m_{k} \ll p_{\text {eff }} \sim 100$ $\mathrm{MeV}$ ), given by

$$
A_{L} \propto \sum_{k}^{\text {light }} m_{k} U_{e k}^{2} M^{0 \nu 2 \beta}\left(m_{k}\right) \simeq m_{e e} M^{0 \nu 2 \beta}(0),
$$

with $M^{0 \nu 2 \beta}(0) \propto 1 / p_{\text {eff }}^{2}$ the nuclear matrix element. The cosmology upper bound on the sum of neutrino masses, $\sum_{k} m_{k} \lesssim 1 \mathrm{eV}[59,60]$, combined with neutrino oscillation data, leads to an upper limit on each neutrino mass $m_{k} \lesssim 0.3 \mathrm{eV}$ and on the element of the neutrino mass matrix relevant to $0 \nu 2 \beta$ decay, $m_{e e} \lesssim 0.3 \mathrm{eV}$.

$A_{\text {md }}$ represents the additional, model dependent contribution due to the unknown physics which generates the three light neutrino masses parametrized by the Weinberg operator. We assume that this last term is negligible compared to $A_{L}$, as it is the case if the underlying mechanism for neutrino masses is any of the standard three see-saw types [61].

We focus then on the contribution from the fourth-generation neutrinos $\left(\nu_{4}, \nu_{\overline{4}}\right)$, given by

$$
\begin{aligned}
A_{4} & \propto N_{e}^{2}\left(m_{4} \cos ^{2} \theta M^{0 \nu 2 \beta}\left(m_{4}\right)-m_{\overline{4}} \sin ^{2} \theta M^{0 \nu 2 \beta}\left(m_{\overline{4}}\right)\right) \\
& \propto N_{e}^{2}\left(\frac{\cos ^{2} \theta}{m_{4}}-\frac{\sin ^{2} \theta}{m_{\overline{4}}}\right)=N_{e}^{2} \frac{m_{\overline{4}}^{2}-m_{4}^{2}}{m_{4} m_{\overline{4}}\left(m_{4}+m_{\overline{4}}\right)}=N_{e}^{2} \frac{m_{\mathrm{R}}}{m_{\mathrm{D}}^{2}},
\end{aligned}
$$

where we have used that $M^{0 \nu 2 \beta}\left(m_{a}\right) \propto 1 / m_{a}^{2}$ for $a=4, \overline{4}, \tan ^{2} \theta=m_{4} / m_{\overline{4}}, m_{4} m_{\overline{4}}=$ $m_{\mathrm{D}}^{2}$ and $m_{\overline{4}}-m_{4}=m_{\mathrm{R}}$. From eqs. (5.4) and (5.5) we see that the fourth-generation neutrino contribution to the $0 \nu 2 \beta$ amplitude can be dominant provided $N_{e}^{2} m_{\mathrm{R}} / m_{\mathrm{D}}^{2}>$ $m_{e e} /(100 \mathrm{MeV})^{2}$. Notice, in fact, that the value of $m_{e e}$ could be zero if normal hierarchy is realised and the neutrino phases have the appropriate values; in this extreme case the only contribution would be that of the fourth generation, which would dominate $0 \nu 2 \beta$.

Now we can exploit the dependence on $N_{e}^{2} m_{\mathrm{R}}$ of both $A_{4}$ and $\left(m_{\nu}\right)_{33}^{(2)}$ in eq. (4.15) to constrain the fourth-generation neutrino contribution to the $0 \nu 2 \beta$ decay amplitude ${ }^{8}$, namely

$$
A_{4} \leq\left(\frac{4 \pi m_{W}}{g m_{\mathrm{D}}}\right)^{4} \frac{2\left(m_{\nu}\right)_{33}^{(2)}}{m_{E}^{2} \ln \frac{m_{E}}{m_{\overline{4}}}} \lesssim 190\left(m_{\nu}\right)_{33}^{(2)}\left(\frac{50 \mathrm{GeV}}{m_{\mathrm{D}}}\right)^{4} \mathrm{GeV}^{-1},
$$

where we have taken into account the LEP limit, $m_{E} \gtrsim 100 \mathrm{GeV}$ and set $\ln \left(m_{E} / m_{\overline{4}}\right) \simeq$ 1. From this equation, it is clear that the largest fourth-generation contributions to the amplitude $A_{4}$ correspond to a small Dirac neutrino mass, $m_{\mathrm{D}}$. Imposing that the twoloop mass matrix element $\left(m_{\nu}\right)_{33}^{(2)}$ is below the cosmology upper bound, $0.3 \mathrm{eV}$, we obtain $A_{4}<6 \times 10^{-8}\left(50 \mathrm{GeV} / m_{\mathrm{D}}\right)^{4} \mathrm{GeV}^{-1}$, while if we require that the two-loop contribution is at most the atmospheric mass scale, $0.05 \mathrm{eV}$, we find $A_{4}<10^{-8}\left(50 \mathrm{GeV} / m_{\mathrm{D}}\right)^{4} \mathrm{GeV}^{-1}$. On the other hand, the non-observation of $0 \nu 2 \beta$ implies that $A_{4}<10^{-8} \mathrm{GeV}^{-1}$ [62], while future

\footnotetext{
${ }^{8}$ Note that $\left(m_{\nu}\right)_{33}^{(2)}$ receives contributions from $N_{e}, N_{\mu}$ and $N_{\tau}$, while $0 \nu 2 \beta$ only involves $N_{e}$.
} 
sensitivity is expected to improve this limit one order of magnitude. Bringing these two results together, we see that, once the constraint from light neutrino masses is taken into account, the contribution of the fourth-generation neutrinos to the $0 \nu 2 \beta$ decay amplitude can reach observable values only in the small region of parameter space $m_{\mathrm{D}} \lesssim 100 \mathrm{GeV}$ (see figure 4), even though it is the dominant one for a larger set of allowed masses and mixings.

\subsection{Four generations and the Higgs boson}

It is well known that due to the presence of a new generation there is an enhancement of the Higgs-gluon-gluon vertex, which arises from a triangle diagram with all quarks running in the loop. This vertex is enhanced approximately by a factor 3 in the presence of a heavy fourth generation, therefore the Higgs production cross section through gluon fusion at the LHC is enhanced by a factor of 9 . However, Higgs decay channels are also strongly modified, in particular the Higgs to gluon decays are equally enhanced, while the $\gamma \gamma$ channel is reduced because of a cancellation between the quark and $W$ contributions. Moreover some of these channels, $\gamma \gamma$ for instance, suffer from important electroweak radiative corrections [63].

With the first LHC data, ATLAS and CMS ruled out at 95\% C.L the range $120-$ $600 \mathrm{GeV}$ for a SM4 Higgs boson, assuming very large masses for the fourth-generation particles [64]. However, different authors have noticed that if fourth-generation neutrinos are light enough $\left(m_{W} / 2 \lesssim m_{\nu_{4}} \lesssim m_{W}\right)$, the decay mode of the Higgs into fourth-generation neutrinos can be dominant for $m_{H} \lesssim 2 m_{W}$ [65-68]. Moreover, if the lightest fourthgeneration neutrino is long-lived this decay channel is invisible and the excluded range for the SM4 Higgs boson is reduced to $160-500 \mathrm{GeV}[67,68]$. In general, if the fourthgeneration neutrinos have both Dirac $\left(m_{\mathrm{D}}\right)$ and Majorana $\left(m_{\mathrm{R}}\right)$ masses, the Higgs can decay to more channels: $\nu_{4} \nu_{4}, \nu_{\overline{4}} \nu_{\overline{4}}$ and $\nu_{4} \nu_{\overline{4}}$.

Recently, ATLAS and CMS have analysed new data, including more Higgs decay channels $[69,70]$, and they have a preliminary low-mass $(\sim 125 \mathrm{GeV})$ hint of the Higgs boson in several channels ${ }^{9}$. In particular there is an excess in the $\gamma \gamma$ channel with respect to the SM3 prediction. For such a light Higgs, the expected ratio of number of events into $\gamma \gamma$ for SM4 over SM3 is about $1.5-2.5$ at leading order [72,73]. However, a global fit to all relevant observables (Higgs searches and electroweak precision data), assuming Dirac neutrinos and a Higgs mass of $125 \mathrm{GeV}$, shows that data are better described by the SM3 [74]. On the other hand, as commented above, within the SM4 the cancellations in the $\gamma \gamma$ channel at leading order render next-to-leading order radiative corrections important. These corrections tend to decrease even further the two-photon production rate $\sigma(g g \rightarrow H) \times\left. B R(H \rightarrow \gamma \gamma)\right|_{S M 4}$. Therefore, were the $125 \mathrm{GeV}$ Higgs hint confirmed, by combining the $\gamma \gamma, Z Z^{*}, W W^{*}$ and the $f \bar{f}$ channels a perturbative SM4 with just one $S M$ Higgs doublet would be excluded, even in the case $m_{\nu_{4}}<m_{W}[75,76]$. Otherwise, in principle it seems possible that if $m_{\nu_{4}} \lesssim m_{W}$ and $\nu_{4}$ is long-lived, some portion of the

\footnotetext{
${ }^{9}$ Also Fermilab CDF and D0 have presented some preliminary results pointing to some excess around this mass which can be assigned mainly to $H \rightarrow b \bar{b}$ decays in $H W$ and $H Z$ associated production [71].
} 
low Higgs mass parameter space, previously allowed to be between $114-160 \mathrm{GeV}$, is still allowed by the new data. Moreover, if one does not trust the convergence of perturbation theory in the $\gamma \gamma$ channel and drops it from the global analysis, including Higgs searches, $R_{b}$ and oblique parameters, the SM4 with Dirac neutrinos is strongly constrained but still viable [77]. Considering neutrino Majorana masses will presumably open up even more the allowed parameter space of the model.

The previous bounds from LEP on the masses of unstable (in collider sense) fourthgeneration neutrinos were $m_{\nu_{4}}>62.1 \mathrm{GeV}$. Using CDF inclusive like-sign dilepton analysis, $\nu_{4}$ masses below $m_{W}$ can be excluded for Higgs masses up to $2 m_{W}$ [67], therefore in this case the ATLAS and CMS analysis for the Higgs boson still apply, and at least the range $120-600 \mathrm{GeV}$ for a SM4 Higgs boson is excluded.

To know definitely whether the SM4 Higgs boson is excluded or not, we will have to wait for new data and a combined analysis of the different channels, $\gamma \gamma, Z Z^{*}, W W^{*}$ and $f \bar{f}$, including correctly all radiative corrections. However, even if the SM-like four-generation Higgs is excluded, many possibilities may arise in extensions of a four-generation scenario, for instance, with an extra Higgs doublet (see $[78,79]$ where the observed signatures of LHC are explained in the framework of $4 \mathrm{G}$ two-Higgs-doublet models).

\section{Conclusions}

We have addressed the question of the generation and nature of neutrino masses in the context of the SM with four families of quarks and leptons.

The three light neutrinos can obtain their masses from a variety of mechanisms with or without new neutral fermions, but the huge hierarchy among such masses and those of the remaining fermions is more naturally explained assuming that they have Majorana nature.

On the other hand, current bounds on fourth-generation neutrino masses imply that, although in principle the same mechanisms are also available, most of them are not natural or provide too small fourth-generation neutrino masses; therefore, we have argued that at least one right-handed neutrino is needed. This would suggest that, contrary to the light neutrinos, fourth-generation ones are naturally Dirac.

However, we have shown that if lepton number is not conserved in the light neutrino sector, the right-handed neutrino must have a Majorana mass term whose size depends on the underlying mechanism for LNV, unless Yukawa couplings of the light leptons to the right-handed neutrino are forbidden. We have estimated the natural size of such Majorana mass term within two frameworks for the light neutrino masses, namely see-saw type I and type II. We have seen that, even if we set it to zero by hand in the Lagrangian at tree level, it is generated at two-loops, and although it depends on the Yukawa couplings and the LNV scale responsible for light neutrino masses, it can be up to the TeV scale. We have developed a model where this Majorana mass is forbidden at tree level by a global symmetry, and it is generated radiatively and finite once this symmetry is broken spontaneously (see appendix A). 
We have then considered a minimal four-generation scenario, with neutrino Majorana masses parametrized by the Weinberg operator and one right-handed neutrino $\nu_{\mathrm{R}}$, which has Yukawa couplings to the four lepton doublets and non-zero Majorana mass. We have analyzed the phenomenological constraints on the parameter space of such a model, derived from direct searches for four-generation leptons, universality tests, charged lepton flavour-violating processes and neutrinoless double beta decay. We have pointed out that the Majorana mass for the fourth-generation neutrino induces relatively large two-loop contributions to the light neutrino masses, which can easily exceed the atmospheric scale and the cosmological bounds. Indeed, this sets the strongest limits on the masses and mixings of fourth-generation neutrinos, collected in figure 6 .

To summarize, in the context of a SM with four generations, we have shown that if light neutrinos are Majorana particles, it is natural that also the fourth-generation neutrino has the Majorana character. We did so by calculating the fourth-neutrino Majorana masses induced by the three light neutrino ones. This has important implications for the neutrino and Higgs sectors of these models, which are being actively tested at the LHC.

\section{Acknowledgments}

We thank Enrique Fernández-Martínez and Jacobo López-Pavón for fruitful discussions on the matter of neutrinoless double beta decay. This work has been partially supported by the Spanish MICINN under grants FPA-2007-60323, FPA-2008-03373, FPA2011-23897, FPA2011-29678-C02-01, Consolider-Ingenio PAU (CSD2007-00060) and CPAN (CSD200700042) and by Generalitat Valenciana grants PROMETEO/2009/116 and PROMETEO/2009/128. A.A. and J.H.-G. are supported by the MICINN under the FPU program.

\section{A. A model for calculable right-handed neutrino masses}

In this appendix we present a model which gives a realistic pattern of neutrino masses in the context of the SM with four-generations and in which the right-handed neutrino mass of the fourth generation is generated radiatively and finite. This is an illustration of the general (model-independent) mechanism discussed in section 3 which allowed us to estimate the size of Majorana neutrino masses for the fourth-generation right-handed neutrinos if the three light active neutrinos are Majorana particles.

Let us consider the SM with four generations and four right-handed neutrinos $\nu_{\mathrm{R} i}$ $(i=1, \cdots, 4)$. To implement the ordinary see-saw, we need three of them very heavy while one of them should be much lighter in order to avoid a too light fourth-generation active neutrino. Then, it is natural to require that one of the fourth right-handed neutrino is massless at tree level and let its mass be generated by radiative corrections. For that purpose we add three extra chiral singlets $s_{\mathrm{L} a}(a=1, \cdots, 3)$. In order to break lepton number we will also include a complex scalar singlet $\sigma$

We assign lepton number in the following way

$$
\ell_{j} \rightarrow e^{i \alpha} \ell_{j}, \quad e_{\mathrm{R} j} \rightarrow e^{i \alpha} e_{\mathrm{R} j}, \quad \nu_{\mathrm{R} j} \rightarrow e^{i \alpha} \nu_{\mathrm{R} j}, \quad \sigma \rightarrow e^{i \alpha} \sigma
$$


the $s_{\mathrm{L} a}$ do not carry lepton number. With these assignments and the requirement that lepton number is conserved we have the following Yukawa Lagrangian

$$
\mathcal{L}_{Y}=-\bar{\ell} Y_{e} e_{\mathrm{R}} \phi-\bar{\ell} Y_{\nu} \nu_{\mathrm{R}} \tilde{\phi}-\sigma \overline{\nu_{\mathrm{R}}} y^{*} s_{\mathrm{L}}-\frac{1}{2} \overline{s_{\mathrm{L}}^{\mathrm{c}}} M^{*} s_{\mathrm{L}}+\text { H.c. }
$$

where $Y_{e}$ and $Y_{\nu}$ are the ordinary four-generation Yukawa couplings, $y_{i a}$, along this appendix, is a general $4 \times 3$ matrix while $M$ is a symmetric $3 \times 3$ matrix, which without loss of generality can be taken diagonal and positive. We choose the scalar potential in such a way that lepton number is conserved and subsequently spontaneously broken by the VEV of $\sigma, v_{\sigma}=\langle\sigma\rangle$. Thus, the model will contain a singlet Majoron. Alternatively, we could also choose to softly break lepton number in the potential to avoid the Majoron without changing the point we would like to illustrate. Before spontaneous symmetry breaking only $s_{\mathrm{L} a}$ are massive. We will take $M$ very large (around GUT scale). After $\sigma$ gets a VEV (which is somewhat free, but we can take it just a bit below $M$ ), we will have a mass matrix for the combined system $\nu_{\mathrm{R}}-s_{\mathrm{L}}$ of see-saw type. Therefore, if $y v_{\sigma} \ll M$ the four right-handed neutrinos will get a $4 \times 4$ Majorana mass matrix

$$
M_{\mathrm{R}}^{(0)} \simeq v_{\sigma}^{2} y M^{-1} y^{\mathrm{T}}
$$

this is basically the see-saw formula but applied to the right-handed neutrinos and changing the VEV of the Higgs doublet for that of the singlet $\sigma$. This matrix has rank 3 and, therefore, only three of the right-handed neutrinos will obtain a tree-level mass. The other neutrino will remain massless at tree level. However, at two loops, due to the mechanism described in section 3, also the fourth right-handed neutrino will acquire a Majorana mass. We depict the diagram giving rise to this mass in figure 7 ; the diagram is obviously finite by power counting and the generated mass matrix can be estimated as

$$
M_{\mathrm{R}}^{(2)} \sim \frac{v_{\sigma}^{2}}{(4 \pi)^{4}}\left(Y_{\nu}^{\dagger} Y_{\nu}\right)^{\mathrm{T}} y M^{-1} y^{\mathrm{T}} Y_{\nu}^{\dagger} Y_{\nu} \ln \left(\frac{M}{y v_{\sigma}}\right)
$$

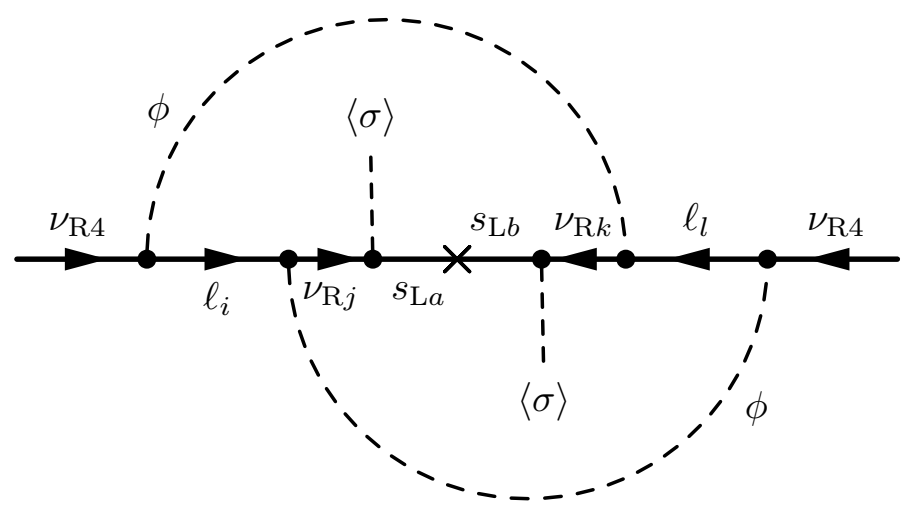

Figure 7: The process which generates masses for the right-handed neutrinos at two loops.

Since $Y_{e}$ does not enter in these calculations we can choose a basis in which $Y_{e}$ is arbitrary but $Y_{\nu}$ is diagonal and real. If we take the logarithm order $1, \ln \left(\frac{M}{y v_{\sigma}}\right) \sim 1$, we 
see that $M_{\mathrm{R}}^{(2)}$ is also projective but in a different direction, given by $y^{\prime}=\left(Y_{\nu}^{\dagger} Y_{\nu}\right)^{\mathrm{T}} y$; then we can write the full right-handed neutrino mass matrix as

$$
M_{\mathrm{R}} \sim v_{\sigma}^{2}\left(y M^{-1} y^{\mathrm{T}}+\frac{1}{(4 \pi)^{4}} y^{\prime} M^{-1} y^{\prime \mathrm{T}}\right),
$$

which, in general, has rank 4 and gives a Majorana mass to the fourth right-handed neutrino. To see how it works, let us discuss a simplified example, with the following structure for the $s_{\mathrm{L}}$ Yukawas:

$$
y=\left(\begin{array}{ccc}
y_{1} & 0 & 0 \\
0 & y_{2} & 0 \\
0 & 0 & y_{3} \\
0 & 0 & y_{4}
\end{array}\right)
$$

Let us also choose $M$ diagonal and with elements $M_{i}$; then, at tree level we obtain an almost diagonal mass matrix,

$$
M_{\mathrm{R}}^{(0)}=v_{\sigma}^{2}\left(\begin{array}{cccc}
\frac{y_{1}^{2}}{M_{1}} & 0 & 0 & 0 \\
0 & \frac{y_{2}^{2}}{M_{2}} & 0 & 0 \\
0 & 0 & \frac{y_{3}^{2}}{M_{3}} & \frac{y_{3} y_{4}}{M_{3}} \\
0 & 0 & \frac{y_{3} y_{4}}{M_{3}} & \frac{y_{4}^{2}}{M_{3}}
\end{array}\right),
$$

which has a zero eigenvalue. At two loops we will have

$$
M_{\mathrm{R}}^{(2)}=\frac{v_{\sigma}^{2}}{(4 \pi)^{4}}\left(\begin{array}{cccc}
\frac{y_{1}^{\prime 2}}{M_{1}} & 0 & 0 & 0 \\
0 & \frac{y_{2}^{\prime 2}}{M_{2}} & 0 & 0 \\
0 & 0 & \frac{y_{3}^{\prime 2}}{M_{3}} & \frac{y_{3}^{\prime} y_{4}^{\prime}}{M_{3}} \\
0 & 0 & \frac{y_{3}^{\prime} y_{4}^{\prime}}{M_{3}} & \frac{y_{4}^{\prime 2}}{M_{3}}
\end{array}\right),
$$

with $y_{i}^{\prime}=y_{i}\left(Y_{\nu}\right)_{i}{ }^{2}$, and $\left(Y_{\nu}\right)_{i}$ the diagonal elements of $Y_{\nu} . M_{\mathrm{R}}^{(2)}$ has also rank 3. However, the sum of $M_{\mathrm{R}}^{(0)}$ and $M_{\mathrm{R}}^{(2)}$ has rank 4, and the fourth $\nu_{\mathrm{R}}$ acquires a mass. We can estimate it by considering $M_{\mathrm{R}}^{(2)}$ a small perturbation to $M_{\mathrm{R}}^{(0)}$ and find that

$$
m_{\mathrm{R} 4} \sim \frac{v_{\sigma}^{2}}{(4 \pi)^{4} M_{3}} \frac{y_{4}^{2} y_{3}^{2}}{y_{3}^{2}+y_{4}^{2}}\left(\left(Y_{\nu}\right)_{4}^{2}-\left(Y_{\nu}\right)_{3}^{2}\right)^{2},
$$

while the mass of the third right-handed neutrino is of order (the other two are also order $y^{2} v_{\sigma}^{2} / M$ as can be seen from the mass matrix)

$$
m_{\mathrm{R} 3} \sim\left(y_{3}^{2}+y_{4}^{2}\right) \frac{v_{\sigma}^{2}}{M_{3}} .
$$

Therefore, if we rewrite the fourth-generation right-handed neutrino mass $m_{\mathrm{R} 4}$ in terms of $m_{\mathrm{R} 3}$ we have

$$
m_{\mathrm{R} 4} \sim \frac{m_{\mathrm{R} 3}}{(4 \pi)^{4}} \frac{y_{4}^{2} y_{3}^{2}}{\left(y_{3}^{2}+y_{4}^{2}\right)^{2}}\left(\left(Y_{\nu}\right)_{4}^{2}-\left(Y_{\nu}\right)_{3}^{2}\right)^{2}
$$


which is roughly the structure that one would expect from the effective theory obtained by integrating the new fermions $s_{\mathrm{L} a}$, i.e, $m_{\mathrm{R} 4}$ obtains a contribution proportional to the heavy right-handed Majorana masses $m_{\mathrm{R} 3}$ suppressed by a two-loop factor and Yukawa couplings. After all, the diagram in figure 7 reduces to the diagram in figure 1 when the fermion lines of $s_{\mathrm{L} a}$ are contracted to a point. The result also shows that, as expected, the exact coefficient depends on the details of the model. These expressions could be generalized to a more general structure of Yukawa couplings, leading to similar, although more complicated expressions.

As for other features of this model, we will just mention that as lepton number is broken spontaneously, a Majoron will appear. Since the Majoron is a singlet and $v_{\sigma}$ is large their couplings to standard model particles are suppressed and, therefore, this Majoron should not create any problem. On the other hand, it could have some advantages in cosmological contexts; if lepton number is also broken softly (for instance with a mass term $\sigma^{2}$ ) the Majoron will become a massive pseudo-Majoron, which could constitute a good dark matter candidate.

In any case, this simple example illustrates how the general mechanism discussed in section 3 works in a complete renormalizable model; if $m_{\mathrm{R} 4}$ is zero at tree level and light neutrinos are Majorana (therefore lepton number is not conserved), in general $m_{\mathrm{R} 4}$ will be generated at two-loops with the behaviour discussed in section 3 .

\section{References}

[1] B. Holdom, W. Hou, T. Hurth, M. Mangano, S. Sultansoy, et al., Four Statements about the Fourth Generation, PMC Phys. A3 (2009) 4, [arXiv:0904.4698] [InSPIRE].

[2] W.-S. Hou, Source of CP Violation for the Baryon Asymmetry of the Universe, Chin.J.Phys. 47 (2009) 134, [arXiv: 0803.1234] [InSPIRE].

[3] J. I. Silva-Marcos, Symmetries, large leptonic mixing and a fourth generation, JHEP 0212 (2002) 036, [arXiv:hep-ph/0204217] [InSPIRE].

[4] Particle Data Group Collaboration, K. Nakamura et al., Review of particle physics, J.Phys.G G37 (2010) 075021 [InSPIRE].

[5] H.-J. He, N. Polonsky, and S.-f. Su, Extra families, Higgs spectrum and oblique corrections, Phys.Rev. D64 (2001) 053004, [arXiv:hep-ph/0102144] [InSPIRE].

[6] V. Novikov, A. Rozanov, and M. Vysotsky, Once more on extra quark-lepton generations and precision measurements, Phys.Atom.Nucl. 73 (2010) 636-642, [arXiv:0904.4570] [InSPIRE]. Dedicated to L.B. Okun's 80th birthday.

[7] M. Gonzalez-Garcia and M. Maltoni, Phenomenology with Massive Neutrinos, Phys.Rept. 460 (2008) 1-129, [arXiv:0704.1800] [InSPIRE].

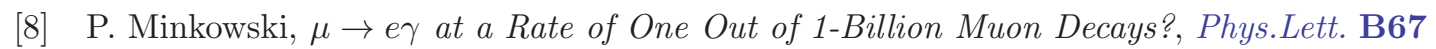
(1977) 421 [InSPIRE].

[9] M. Gell-Mann, P. Ramond, and R. Slansky, Complex Spinors and Unified Theories, Conf.Proc. C790927 (1979) 315-321 [InSPIRE]. Published in Supergravity, P. van Nieuwenhuizen and D.Z. Freedman (eds.), North Holland Publ. Co., 1979. 
[10] T. Yanagida, Horizontal Symmetry and Masses of Neutrinos, Conf.Proc. C7902131 (1979) 95 [InSPIRE].

[11] R. N. Mohapatra and G. Senjanovic, Neutrino Mass and Spontaneous Parity Violation, Phys.Rev.Lett. 44 (1980) 912 [InSPIRE].

[12] A. Zee, A Theory of Lepton Number Violation, Neutrino Majorana Mass, and Oscillation, Phys.Lett. B93 (1980) 389 [InSPIRE].

[13] A. Zee, Quantum Numbers of Majorana Neutrino Masses, Nucl.Phys. B264 (1986) 99 [InSPIRE].

[14] K. Babu, Model of 'Calculable' Majorana Neutrino Masses, Phys.Lett. B203 (1988) 132 [InSPIRE].

[15] E. Ma, Verifiable radiative seesaw mechanism of neutrino mass and dark matter, Phys.Rev. D73 (2006) 077301, [arXiv:hep-ph/0601225] [InSPIRE].

[16] K. Babu and E. Ma, Natural Hierarchy of Radiatively Induced Majorana Neutrino Masses, Phys.Rev.Lett. 61 (1988) 674 [InSPIRE].

[17] K. Babu, E. Ma, and J. T. Pantaleone, Model of Radiative Neutrino Masses: Mixing and a Possible Fourth Generation, Phys.Lett. B218 (1989) 233-237 [InSPIRE].

[18] C. Hill and E. Paschos, A Naturally Heavy Fourth Generation Neutrino, Phys.Lett. B241 (1990) 96 [InSPIRE].

[19] R. Mohapatra and X. Zhang, Restrictions on B-L symmetry breaking implied by a fourth generation neutrino, Phys.Lett. B305 (1993) 106-108, [arXiv:hep-ph/9301286] [InSPIRE].

[20] L. M. Carpenter and A. Rajaraman, Revisiting Constraints on Fourth Generation Neutrino Masses, Phys.Rev. D82 (2010) 114019, [arXiv:1005.0628] [InSPIRE].

[21] A. Rajaraman and D. Whiteson, Tevatron Discovery Potential for Fourth Generation Neutrinos: Dirac, Majorana and Everything in Between, Phys.Rev. D82 (2010) 051702, [arXiv: 1005.4407] [InSPIRE].

[22] A. Lenz, H. Pas, and D. Schalla, Constraints on fourth generation Majorana neutrinos, J.Phys.Conf.Ser. 259 (2010) 012096, [arXiv: 1010.3883] [InSPIRE].

[23] A. Aparici, J. Herrero-Garcia, N. Rius, and A. Santamaria, Neutrino masses from new generations, JHEP 1107 (2011) 122, [arXiv:1104.4068] [InSPIRE].

[24] A. Lenz, H. Pas, and D. Schalla, Fourth Generation Majorana Neutrinos, Phys.Rev. D85 (2012) 075025, [arXiv:1104.2465] [InSPIRE].

[25] M. A. Schmidt and A. Y. Smirnov, Neutrino Masses and a Fourth Generation of Fermions, Nucl.Phys. B857 (2012) 1-27, [arXiv:1110.0874] [InSPIRE].

[26] A. Aparici, J. Herrero-Garcia, N. Rius, and A. Santamaria, Implications of new generations on neutrino masses, [arXiv:1110.0663] [InSPIRE]. Published in NUFACT 2011, XIIIth International Workshop on Neutrino Factories, Super beams and Beta beams, 1-6 August 2011, CERN and University of Geneva.

[27] S. Petcov and S. Toshev, Conservation of Lepton Charges, Massive Majorana and Massless Neutrinos, Phys.Lett. B143 (1984) 175 [InSPIRE].

[28] G. Branco, W. Grimus, and L. Lavoura, The Seesaw Mechanism in the Presence of a Conserved Lepton Number, Nucl.Phys. B312 (1989) 492 [InSPIRE]. 
[29] W. Grimus and H. Neufeld, Radiative Neutrino Masses in an Su(2) X U(1) Model, Nucl.Phys. B325 (1989) 18 [InSPIRE].

[30] S. Weinberg, Baryon and Lepton Nonconserving Processes, Phys.Rev.Lett. 43 (1979) 1566-1570 [InSPIRE].

[31] H. Weldon and A. Zee, Operator Analysis of New Physics, Nucl.Phys. B173 (1980) 269 [InSPIRE].

[32] P. Ramond, The Family Group in Grand Unified Theories, [arXiv:hep-ph/9809459] [InSPIRE]. Retro-Preprint (1979 unpublished Caltech preprint no: CALT-68-709). Invited talk at the Sanibel Symposia, Feb 1979.

[33] W. Konetschny and W. Kummer, Nonconservation of Total Lepton Number with Scalar Bosons, Phys.Lett. B70 (1977) 433 [InSPIRE].

[34] T. Cheng and L.-F. Li, Neutrino Masses, Mixings and Oscillations in SU(2) x U(1) Models of Electroweak Interactions, Phys.Rev. D22 (1980) 2860 [InSPIRE].

[35] G. Lazarides, Q. Shafi, and C. Wetterich, Proton Lifetime and Fermion Masses in an SO(10) Model, Nucl.Phys. B181 (1981) 287 [InSPIRE].

[36] M. Magg and C. Wetterich, Neutrino Mass Problem and Gauge Hierarchy, Phys.Lett. B94 (1980) 61 [InSPIRE].

[37] J. Schechter and J. Valle, Neutrino Masses in SU(2) x U(1) Theories, Phys.Rev. D22 (1980) 2227 [InSPIRE].

[38] S. Kanemura and K. Yagyu, Radiative corrections to electroweak parameters in the $Y=1$ Higgs triplet model and implication with the recent Higgs boson searches at the CERN LHC, [arXiv:1201.6287] [InSPIRE].

[39] F. del Aguila, J. Aguilar-Saavedra, J. . de Blas, and M. Perez-Victoria, Electroweak constraints on see-saw messengers and their implications for LHC, [arXiv:0806.1023] [InSPIRE]. Published in the Proceedings of "43rd Rencontres de Moriond on Electroweak Interactions and Unified Theories", 1-8 Mar 2008, La Thuile, Italy.

[40] R. Foot, H. Lew, X. He, and G. C. Joshi, Seesaw Neutrino Masses Induced by a Triplet of Leptons, Z.Phys. C44 (1989) 441 [InSPIRE].

[41] E. Ma and D. Roy, Heavy triplet leptons and new gauge boson, Nucl.Phys. B644 (2002) 290-302, [arXiv:hep-ph/0206150] [InSPIRE].

[42] C. Aulakh and R. N. Mohapatra, Neutrino as the Supersymmetric Partner of the Majoron, Phys.Lett. B119 (1982) 136 [InSPIRE].

[43] L. J. Hall and M. Suzuki, Explicit R-Parity Breaking in Supersymmetric Models, Nucl.Phys. B231 (1984) 419 [InSPIRE].

[44] I.-H. Lee, Lepton Number Violation in Softly Broken Supersymmetry, Phys.Lett. B138 (1984) 121 [InSPIRE].

[45] I.-H. Lee, Lepton Number Violation in Softly Broken Supersymmetry. 2., Nucl.Phys. B246 (1984) 120 [InSPIRE].

[46] J. R. Ellis, G. Gelmini, C. Jarlskog, G. G. Ross, and J. Valle, Phenomenology of Supersymmetry with Broken R-Parity, Phys.Lett. B150 (1985) 142 [InSPIRE]. 
[47] G. G. Ross and J. Valle, Supersymmetric Models Without R-Parity, Phys.Lett. B151 (1985) 375 [InSPIRE].

[48] S. Dawson, R-Parity Breaking in Supersymmetric Theories, Nucl.Phys. B261 (1985) 297 [InSPIRE].

[49] A. Santamaria and J. Valle, Spontaneous R-Parity Violation in Supersymmetry: A Model for Solar Neutrino Oscillations, Phys.Lett. B195 (1987) 423 [InSPIRE].

[50] A. Masiero and J. Valle, A Model for Spontaneous R Parity Breaking, Phys.Lett. B251 (1990) 273-278 [InSPIRE].

[51] M. Hirsch, M. Diaz, W. Porod, J. Romao, and J. Valle, Neutrino masses and mixings from supersymmetry with bilinear $R$ parity violation: A Theory for solar and atmospheric neutrino oscillations, Phys.Rev. D62 (2000) 113008, [arXiv:hep-ph/0004115] [InSPIRE].

[52] R. Barbier, C. Berat, M. Besancon, M. Chemtob, A. Deandrea, et al., R-parity violating supersymmetry, Phys.Rept. 420 (2005) 1-202, [arXiv:hep-ph/0406039] [InSPIRE].

[53] A. J. Buras, B. Duling, T. Feldmann, T. Heidsieck, and C. Promberger, Lepton Flavour Violation in the Presence of a Fourth Generation of Quarks and Leptons, JHEP 1009 (2010) 104, [arXiv: 1006.5356] [InSPIRE].

[54] L. M. Carpenter, Fourth Generation Lepton Sectors with Stable Majorana Neutrinos: From LEP to LHC, [arXiv:1010.5502] [InSPIRE].

[55] COMET Collaboration Collaboration, E. V. Hungerford, COMET/PRISM muon to electron conversion at J-PARC, AIP Conf.Proc. 1182 (2009) 694-697 [InSPIRE].

[56] MEG Collaboration, J. Adam et al., New limit on the lepton-flavour violating decay $\mu^{+} \rightarrow e^{+} \gamma$, Phys.Rev.Lett. 107 (2011) 171801, [arXiv:1107.5547] [InSPIRE].

[57] S. Antusch, C. Biggio, E. Fernandez-Martinez, M. Gavela, and J. Lopez-Pavon, Unitarity of the Leptonic Mixing Matrix, JHEP 0610 (2006) 084, [arXiv:hep-ph/0607020] [InSPIRE].

[58] A. Pich, Lepton universality, NATO Adv.Study Inst.Ser.B Phys. 363 (1997) 173-190, [arXiv:hep-ph/9701263] [InSPIRE].

[59] S. Hannestad, A. Mirizzi, G. G. Raffelt, and Y. Y. Wong, Neutrino and axion hot dark matter bounds after WMAP-7, JCAP 1008 (2010) 001, [arXiv: 1004.0695] [InSPIRE].

[60] M. Gonzalez-Garcia, M. Maltoni, and J. Salvado, Robust Cosmological Bounds on Neutrinos and their Combination with Oscillation Results, JHEP 1008 (2010) 117, [arXiv:1006.3795] [InSPIRE].

[61] M. Blennow, E. Fernandez-Martinez, J. Lopez-Pavon, and J. Menendez, Neutrinoless double beta decay in seesaw models, JHEP 1007 (2010) 096, [arXiv: 1005.3240] [InSPIRE].

[62] H. Pas, M. Hirsch, H. Klapdor-Kleingrothaus, and S. Kovalenko, A Superformula for neutrinoless double beta decay. 2. The Short range part, Phys.Lett. B498 (2001) 35-39, [arXiv:hep-ph/0008182] [InSPIRE].

[63] A. Denner, S. Dittmaier, A. Muck, G. Passarino, M. Spira, et al., Higgs production and decay with a fourth Standard-Model-like fermion generation, Eur.Phys.J. C72 (2012) 1992, [arXiv: 1111.6395] [InSPIRE].

[64] ATLAS and CMS Collaboration, Combined Standard Model Higgs boson searches with up to $2.3 \mathrm{fb}^{-1}$ of $\mathrm{pp}$ collisions at $\sqrt{\mathrm{s}}=7 \mathrm{TeV}$ at the LHC, Tech. Rep. ATLAS-CONF-2011-157, CERN, Geneva, Nov, 2011. http://cdsweb.cern.ch/record/1399599. 
[65] K. Belotsky, D. Fargion, M. Khlopov, R. Konoplich, and K. Shibaev, Invisible Higgs boson decay into massive neutrinos of fourth generation, Phys.Rev. D68 (2003) 054027, [arXiv:hep-ph/0210153] [InSPIRE].

[66] W.-Y. Keung and P. Schwaller, Long Lived Fourth Generation and the Higgs, JHEP 1106 (2011) 054, [arXiv: 1103.3765] [InSPIRE].

[67] L. M. Carpenter and D. Whiteson, Higgs Decays to Unstable Neutrinos: Collider Constraints from Inclusive Like-Sign Dilepton Searches, [arXiv:1107.2123] [InSPIRE].

[68] S. Cetin, T. Cuhadar-Donszelmann, M. Sahin, S. Sultansoy, and G. Unel, Impact of the relatively light fourth family neutrino on the Higgs boson search, Phys.Lett. B710 (2012) 328-331, [arXiv:1108.4071] [InSPIRE].

[69] ATLAS Collaboration, G. Aad et al., Combined search for the Standard Model Higgs boson using up to $4.9 \mathrm{fb}-1$ of $\mathrm{pp}$ collision data at sqrt(s) $=7 \mathrm{TeV}$ with the ATLAS detector at the LHC, Phys.Lett. B710 (2012) 49-66, [arXiv:1202.1408] [InSPIRE].

[70] CMS Collaboration Collaboration, S. Chatrchyan et al., Combined results of searches for the standard model Higgs boson in pp collisions at sqrt(s) = 7 TeV, Phys.Lett. B710 (2012) 26-48, [arXiv: 1202.1488] [InSPIRE].

[71] TEVNPH (Tevatron New Phenomina and Higgs Working Group), CDF and D0 Collaboration, Combined CDF and DO measurement of WZ and $Z Z$ production in final states with b-tagged jets, [arXiv:1203.3782] [InSPIRE].

[72] G. Guo, B. Ren, and X.-G. He, LHC Evidence Of A $126 \mathrm{GeV}$ Higgs Boson From $H \rightarrow \gamma \gamma$ With Three And Four Generations, [arXiv:1112.3188] [InSPIRE].

[73] X. Ruan and Z. Zhang, Impact on the Higgs Production Cross Section and Decay Branching Fractions of Heavy Quarks and Leptons in a Fourth Generation Model, [arXiv:1105.1634] [InSPIRE].

[74] O. Eberhardt, G. Herbert, H. Lacker, A. Lenz, A. Menzel, et al., Joint analysis of Higgs decays and electroweak precision observables in the Standard Model with a sequential fourth generation, [arXiv:1204.3872] [InSPIRE].

[75] A. Djouadi and A. Lenz, Sealing the fate of a fourth generation of fermions, [arXiv:1204.1252] [InSPIRE].

[76] E. Kuflik, Y. Nir, and T. Volansky, Implications of Higgs Searches on the Four Generation Standard Model, [arXiv:1204.1975] [InSPIRE].

[77] M. Buchkremer, J.-M. Gerard, and F. Maltoni, Closing in on a perturbative fourth generation, JHEP 1206 (2012) 135, [arXiv:1204.5403] [InSPIRE].

[78] N. Chen and H.-J. He, LHC Signatures of Two-Higgs-Doublets with Fourth Family, JHEP 1204 (2012) 062, [arXiv: 1202.3072] [InSPIRE].

[79] L. Bellantoni, J. Erler, J. J. Heckman, and E. Ramirez-Homs, Masses of a Fourth Generation with Two Higgs Doublets, [arXiv:1205.5580] [InSPIRE]. 